\title{
QUISTES DE DINOFLAGELADOS (CLADOPYXIACEAE, GONYAULACACEAE, GONIODOMACEAE E INCIERTA) DEL OLIGOCENO - PLIOCENO TEMPRANO DE LA CUENCA DEL COLORADO, ARGENTINA
}

\author{
M. Verónica GULER $R^{1,2}$ y G. Raquel GUERSTEIN ${ }^{1,2}$ \\ ' Departamento de Geología, Universidad Nacional del Sur. San Juan 670, 8000 Bahía Blanca. \\ Argentina, E- mail: vguler@criba.edu.ar \\ ${ }_{2}^{2}$ Consejo Nacional de Investigaciones Científicas y Técnicas (CONICET).
}

Guler, M.V. y Guerstein, G.R. 2003. Quistes de dinoflagelados (Cladopyxiaceae, Gonyaulacaceae, Goniodomaceae e incierta) del Oligoceno-Plioceno temprano de la cuenca del Colorado, Argentina. [OligoceneEarly Pliocene dinoflagellate cysts (Cladopyxiaceae, Gonyaulacaceae, Goniodomaceae and uncertain) from Colorado Basin, Argentina.] Revista Española de Paleontología, 18(1), 23-47. ISSN 0213-6937.

\begin{abstract}
The aim of this paper is to describe and illustrate the Oligocene to Pliocene dinoflagellate cyst assemblages from Fx-1 and Dx-1 wells and Barranca Final Formation type section. Fx-1 and Dx-1 wells were drilled offshore northern Colorado Basin and the Neogene Barranca Final Formation type section crops out in the southern margin of the basin. The assemblages are diverse and very well preserved. A total of 41 dinoflagellate cyst taxa have been described. 1 species belongs to family Cladopyxiaceae, 29 species to Gonyaulacaceae, 2 species to Goniodomaceae, and 9 species are included in uncertain family. Labyrinthodinium truncatum subsp. truncatum, Cleistosphaeridium ancyreum, Cannosphaeropsis quattrocchiae and other species with biostratigraphically significant last occurrences, were useful for stratigraphical subdivision in the Mid-Cenozoic of the Colorado Basin. Cousteaudinium aubryae, Tectatodinium pellitum, Habibacysta tectata, Bitectatodinium tepikiense, Spiniferites falcipedius are reported for the first time from Argentina. Operculodinium sp. 1, Operculodinium sp. 2, Operculodinium sp. 3, Impagidinium sp. 1, Impagidinium sp. 2, Impagidinium sp. 3., Spiniferites cf. falcipedius and Unipontidinium sp. present distinctive morphologies and are potentially new species.
\end{abstract}

Keywords: Dinoflagellates, Oligocene-Pliocene, Colorado Basin, Argentina.

\section{RESUMEN}

En el presente trabajo se describen e ilustran las asociaciones de quistes de dinoflagelados oligocenas a pliocenas provenientes de los pozos Fx-1, Dx-1 y de la sección tipo de la Formación Barranca Final. Los pozos Fx-1 y Dx1 están ubicados costa afuera en el sector norte de la cuenca del Colorado, y la sección tipo de la Formación Barranca Final aflora sobre el margen sur de la cuenca. Las asociaciones son diversas y presentan muy buena conservación. Se describen 41 taxones de quistes de dinoflagelados, de los cuales 1 especie pertenece a la familia Cladopyxiaceae, 29 a la familia Gonyaulacaceae, 2 a la familia Goniodomaceae, y 9 especies se incluyen en familia incierta. Labyrinthodinium truncatum subsp. truncatum, Cleistosphaeridium ancyreum, Cannosphaeropsis quattrocchiae y otras especies con últimas presencias bioestratigráficamente significativas, permitieron la subdivisión estratigráfica en el Cenozoico medio de la cuenca del Colorado. Cousteaudinium aubryae, Tectatodinium pellitum, Habibacysta tectata, Bitectatodinium tepikiense y Spiniferites falcipedius se registran por primera vez en Argentina. Operculodinium sp. 1, Operculodinium sp. 2, Operculodinium sp. 3, Impagidinium sp. 1, Impagidinium sp. 2, Impagidinium sp. 3, Spiniferites cf. falcipedius y Unipontidinium sp. presentan morfologías distintivas y son potenciales nuevas especies.

Palabras clave: Dinoflagelados, Oligoceno-Plioceno, cuenca del Colorado, Argentina. 


\section{INTRODUCCIÓN}

La cuenca del Colorado está situada en la costa este de Argentina y se desarrolla en su mayor parte sobre la plataforma continental, entre los $38^{\circ}$ y $41^{\circ}$ de latitud Sur y $57^{\circ}$ y $64^{\circ}$ de longitud Oeste. Ocupa la porción sudeste de la Provincia de Buenos Aires y noreste de la Provincia de Río Negro y cubre más de 160.000 kilómetros cuadrados, dos tercios de los cuales yacen costa afuera en aguas de hasta 350 metros de profundidad (Fig. 1). Integra una serie de cuencas de margen Atlántico, que se originaron como parte de un sistema de rift durante el Jurásico Medio a Tardío (Fryklund et al. , 1996).

Las asociaciones de quistes de dinoflagelados (dinoquistes) estudiadas en este trabajo, provienen de las perforaciones Fx-1 $\left(39^{\circ} \mathrm{S} 61^{\circ} 2^{\prime} \mathrm{O}\right)$ y Dx-1 $\left(39^{\circ} \mathrm{S} 59^{\circ} \mathrm{O}\right)$ realizadas por Phillips / AGIP en el sector norte de la cuenca del Colorado y de la sección tipo de la Formación Barranca Final que aflora en la costa del golfo San Matías, en el sudoeste de la cuenca del Colorado (Fig. 1).

Las asociaciones están compuestas predominantemente por especies del orden Gonyaulacales y en menor proporción por especies del orden Peridiniales. Estas últimas están representadas principalmente por la familia Protoperidiniaceae cuyo estudio sistemático fue tratado en Guler (en prensa). La bioestratigrafía detallada de los pozos Fx-1 y Dx-1 fue propuesta en Guler y Guerstein (en prensa). Los datos de últimas presencias de los taxones bioestratigráficamente significativos permitieron diferenciar cinco intervalos: Mioceno tardío a Plioceno temprano, Mioceno tardío, Mioceno medio, Oligoceno tardío a Mioceno temprano y Oligoceno temprano (Fig. 2). Los dinoquistes presentan mayor abundancia y diversidad en los niveles inferiores. En la parte superior de ambos pozos, en depósitos asignados al Mioceno tardío, se observó la desaparición simultánea de un número importante de especies. Este bioevento fue asociado a un episodio de somerización registrado en la cuenca en la mayoría de los pozos previamente estudiados (Gamerro y Archangelsky, 1981; Guerstein y Guler, 2000 y Guerstein y Junciel, 2001 ).

La sección tipo de la Formación Barranca Final es una de las pocas unidades pre-Pleistocenas aflorantes en la cuenca del Colorado. Las asociaciones de quistes de dinoflagelados conjuntamente con las asociaciones de foraminíferos indicaron una edad comprendida entre el Mioceno medio y el Mioceno tardío para toda la sección (Guler et al., 2002).

Las especies descritas en este trabajo corresponden al orden Gonyaulacales y dentro de éste a las familias Cladopyxiaceae, Gonyaulacaceae, Goniodomaceae e Incierta. Las mismas han sido mencionadas e ilustradas previamente en los trabajos bioestratigráficos en la cuenca del Colorado (Gamerro y Archangelsky, 1981; Guerstein y Guler, 2000; Guerstein y Junciel, 2001; Guler y Guerstein, en prensa), en sedimentos asignados al Mioceno correspondientes a la Formación Chenque en la cuenca del golfo San Jorge (Palamarczuk y Barreda, 1998), en depósitos del Oligoceno-Mioceno inferior provenientes del pozo Aries x-1 en la cuenca Austral

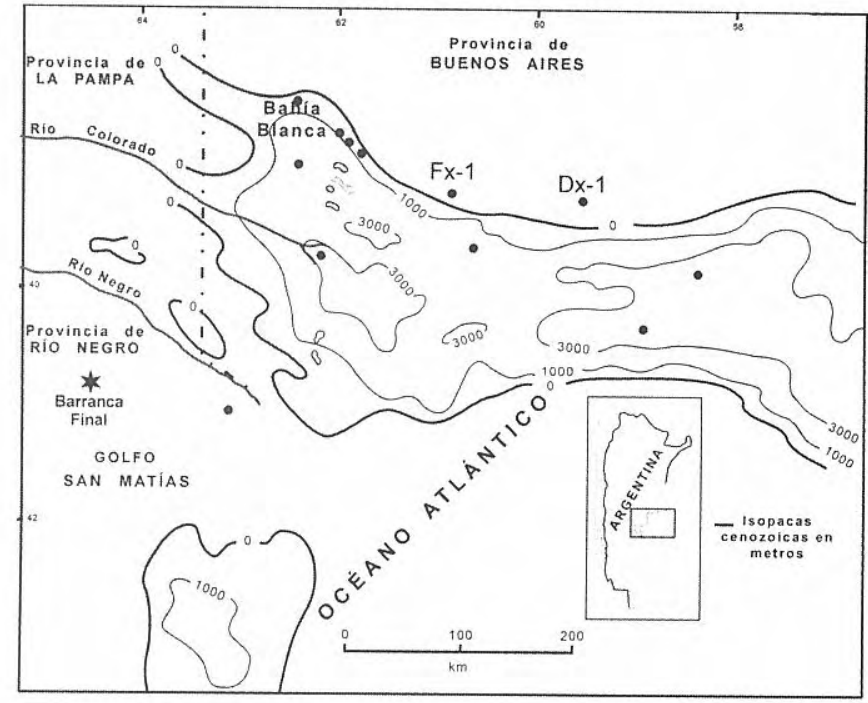

Figura 1. Cuenca del Colorado. Mapa de ubicación de los pozos y localidades estudiadas.

Colorado Basin. Location map showing the wells and outcropping sections.

(Palamarczuk y Barreda, 2000), en localidades a lo largo de la costa de la provincia de Santa Cruz correspondientes a la Formación Monte León (Barreda y Palamarczuk, 2000a) y en depósitos del Oligoceno tardíoMioceno en secciones ubicadas en el área sur del golfo San Jorge (Barreda y Palamarczuk, 2000b).

Los trabajos sistemáticos previos de palinomorfos marinos del Cenozoico medio-tardío en la cuenca del Colorado son escasos. Se mencionan aquellos realizados por Guerstein (1990 a y b) y Guerstein et al. (2001).

\section{MATERIALES Y MÉTODOS}

Se procesaron un total de 51 muestras provenientes de los pozos Dx-1 y Fx-1 y de la sección Barranca Final en el laboratorio de palinología del CIRGEO. Las 23 muestras del pozo Dx-1 están comprendidas entre los 165 y 1120 metros de profundidad con un espaciamiento de 35 metros, de las cuales 9 muestras resultaron palinológicamente estériles. Las 18 muestras del pozo Fx-1 se extienden desde los 365 hasta los 910 metros con un espaciamiento de 20 metros, todas palinológicamente fértiles. Las 10 muestras de afloramiento corresponden a la Formación Barranca Final en su sección tipo. Exhibe 15 metros de arcillas y arcillas arenosas con algunas intercalaciones tobáceas, de color predominantemente grisáceo. Para la concentración de palinomorfos las muestras se trataron con $\mathrm{HCl}, \mathrm{HF}$, se filtraron con mallas de 25 y $10 \mu \mathrm{m}$, fueron teñidas con pardo de Bismarck y montadas en gelatina - glicerina.

Se utilizó un microscopio Nikon Eclipse 600 y las coordenadas de los ejemplares ilustrados corresponden a la escala Vernier. Las fotomicrografías se tomaron con una cámara Nikon H-III con película Ilford 125 ASA. Los preparados están archivados en la Palinoteca del Laboratorio de Palinología de la Universidad Nacional del Sur bajo las siglas UNSLP 999 a 2099 y 4199 a 4399 para el 


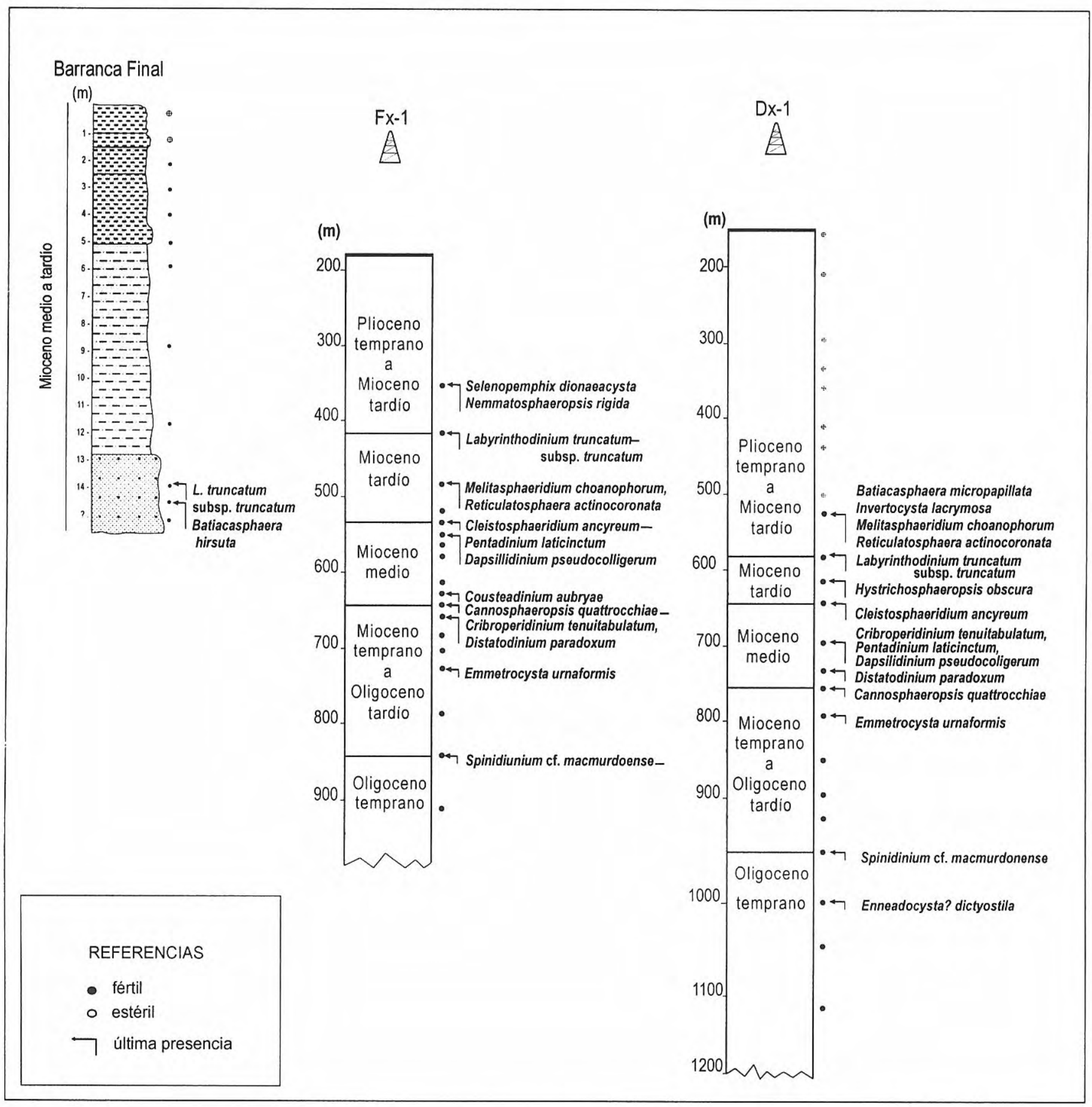

Figura 2. Bioestratigrafía basada en quistes de dinoflagelados de la sección tipo de la Formación Barranca Final y de los pozos Fx-1 y Dx-1 (tomado de Guler et al., 2002 y Guler y Guerstein, en prensa).

Dinoflagellate cyst biostragraphy of Barranca Final type section and Fx-1 and Dx-1 wells (from Guler et al., 2002 and Guler and Guerstein, in press).

pozo DX-1, UNSLP 4499 a 5399 y 0101 a 0801 para el pozo Fx-1 y UNSLP MA21 a ML30 para la sección Barranca Final.

Para la determinación sistemática de las especies de quistes de dinoflagelados se utilizó el índice de quistes de dinoflagelados fósiles de Williams et al., (1998a) y se clasificaron según Fensome et al. (1993) actualizado según Fensome et al. (1998). Las escalas geocronológica y cronoestratigráfica corresponden a Berggren et al. (1995).

\section{SISTEMÁTICA}

DIVISIÓN DINOFLAGELLATA (Butschli 1885) Fensome et al. 1993

SUBDIVISIÓN DINOKARYOTA Fensome et al. 1993

CLASE DINOPHYCEAE Pascher 1914

SUBCLASE PERIDINIPHYCIDAE Fensome et al. 1993

ORDEN GONYAULACALES Taylor 1980

SUBORDEN GONYAULACINAEAE (autonym) 
Familia Cladopyxiaceae Stein 1883

Género Enneadocysta Stover y Williams 1995

Especie tipo: Enneadocysta pectiniformis (Gerlach 1961) Stover y Williams 1995.

\section{Enneadocysta? dictyostila (Menéndez 1965) Stover y Williams 1995 Fig. 3 e}

Dimensiones: Diámetro máximo del cuerpo central, $65 \mu \mathrm{m}$; largo de los procesos, entre 14 y $22 \mu \mathrm{m}$ ( 1 ejemplar medido).

Observaciones: Lentin y Williams (1985) sugieren mantener el nombre Enneadocysta? dictyostila (como Areosphaeridium dictyostilum) para el holotipo solamente. Sin embargo, la observación del material tipo proveniente de Tierra del Fuego permitió asignar el material hallado a esta especie.

Familia Gonyaulacaceae Lindemann 1928

Subfamilia Leptodinioideae Fensome et al. 1993

Género Emmetrocysta (Cookson 1953) Stover 1975

Especie tipo: Emmetrocysta urnaformis (Cookson 1953) Stover 1975.

\section{Emmetrocysta urnaformis \\ (Cookson 1953) Stover 1975}

Fig. 3 f, i, j

Dimensiones: Diámetro del quiste, $40(56,6) 75 \mu \mathrm{m}$; diámetro basal de los procesos, $13(17,6) 21 \mu \mathrm{m}$; largo de los procesos, 15 (15,7) $18 \mu \mathrm{m}$ (9 ejemplares medidos).

Distribución: Pese a que la mayoría de los registros previos son eocenos, cabe destacar que las últimas presencias de esta especie en los pozos estudiados en la cuenca del Colorado alcanzan sedimentos de edad miocena temprana.

Subfamilia Cribroperidinioideae Fensome et al. 1993 Género Cousteaudinium de Verteuil y Norris 1996

Especie tipo: Cousteaudinium aubryae de Verteuil y Norris 1996.

\section{Cousteaudinium aubryae de Verteuil y Norris 1996 \\ Fig. 4 g, h, j - 1}

Dimensiones: Máximo diámetro del endoblasto, $47(49,7)$ $55 \mu \mathrm{m}$; largo de los procesos, $1-18 \mu \mathrm{m}$ (19 ejemplares medidos).

Observaciones: Esta especie fue registrada en sólo dos muestras provenientes de la perforación Fx-1, consideradas de edad serravalianense o más antigua. Los ejemplares hallados presentan marcada similitud con el material tipo del Mioceno de la planicie costera atlántica de Maryland y Virginia. Los procesos alcanzan menor longitud con respecto al material original (1-35 $\mu \mathrm{m})$, coincidiendo con una amplia separación de los blastos. En otras secciones de la cuenca del Colorado ha sido mencionado como $C$. cf. aubryae y constituye el primer registro en el Hemisferio Sur (Guerstein y Junciel, 2001).

Registros previos: Mioceno inferior a Mioceno medio de la planicie costera atlántica de los Estados Unidos (De Verteuil y Norris, 1996).

Género Cribroperidinium Neale y Sarjeant 1962 emend. Davey 1969 emend. Sarjeant 1984 emend. Helenes 1984

Especie tipo: Cribroperidinium sepimentum Neale y Sarjeant 1962.

\section{Cribroperidinium tenuitabulatum (Gerlach 1961) Helenes 1984 Fig. 5 a - c}

Dimensiones: Largo total, $78(89,1) 104 \mu \mathrm{m}$; ancho, 55 $(69,5) 85 \mu \mathrm{m}$ (9 ejemplares medidos).

Observaciones: Los ejemplares medidos superan las dimensiones del material original $(84-93 \mu \mathrm{m})$. El cíngulo mide entre 5 y $7 \mu \mathrm{m}$ y presenta en algunos casos un desplazamiento 3 a 4 veces su ancho.

Distribución estratigráfica: Eoceno Medio (43,5 Ma) Mioceno Medio (13,2 Ma) (Williams et al., 1998 b).

Género Hystrichokolpoma Klumpp 1953

Especie tipo: Hystrichokolpoma cinctum Klumpp 1953.

Figura 3. a - c, Spiniferites falcipedius Warny y Wrenn (x 750) UNSLP 999 39/105, superficie dorsal, dorsal surface; a, foco alto, se observan los extremos de los procesos membranosos, high focus showing membranous processes distal ends; b, foco intermedio, intermediate focus; c, foco bajo, low focus. d, Spiniferites mirabilis (Rossignol) Sarjeant (x 750), UNSLP 2399 27,4/104,5, superficie ventral, foco intermedio, ventral surface, intermediate focus. e, Enneadocysta? dictyostila (Menéndez) Stover y Williams (x 450); superficie ventral, foco alto, ejemplar completo, ventral surface, high focus, complete specimen. $\mathbf{f}, \mathbf{i}, \mathbf{j}$, Emmetrocysta urnaformis (Cookson) Stover, f, (x 750) UNSLP 4299 30,5/103,5, superficie antapical oblicua, foco alto, oblique antapical surface, high focus; i, j, (x 400), UNSLP 4199 39/102, opérculo compuesto por cuatro placas expresadas por los complejos de procesos, opercula composed of four plates indicated by processes complexes; $\mathbf{i}$, foco bajo, low focus; $\mathbf{j}$, foco alto, high focus. $\mathbf{g}, \mathbf{h}, \mathbf{k}, \mathbf{l}$, 

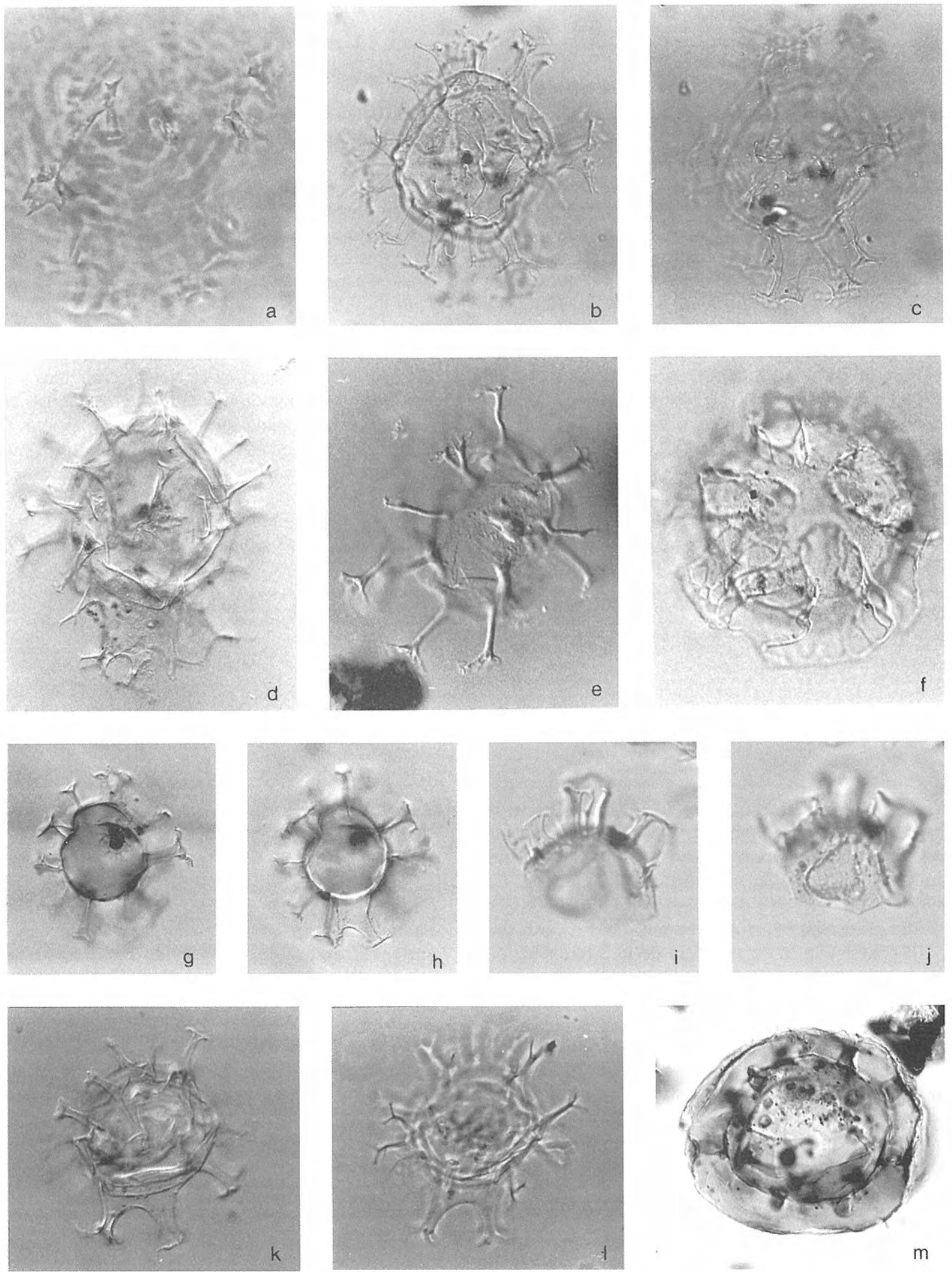

Spiniferites cf. falcipedius Warny y Wrenn (x 500), g, h, UNSLP 2599 27/109,8; g, corte óptico, cross section; h, foco intermedio bajo, low intermediate focus; k, l UNSLP 2599 36,4/110; vista general, general view; k, foco intermedio alto, high intermediate focus; $\mathbf{1}$, foco intermedio bajo, low intermediate focus. m, Tuberculodinium vancampoce (Rossignol) Wall (x 350) UNSLP 2199 35,5/109, superficie antapical, foco alto, antapical surface, high focus. 


\section{Hystrichokolpoma rigaudiae \\ Deflandre y Cookson 1955}

Fig. $6 \mathrm{i}-1$

Dimensiones: Ancho del cuerpo central, $37(45,6) 55 \mu \mathrm{m}$; largo, $35(41,6) 50 \mu \mathrm{m}$; largo de los procesos mayores, 15 $(19,3) 25 \mu \mathrm{m}$; largo del proceso antapical, $23(33,6) 40 \mu \mathrm{m}$ (25 ejemplares medidos).

Distribución: El biocrón de Hystrichokolpoma rigaudiae se extiende hasta el Pleistoceno (Williams et al., 1998b). Sin embargo, en los pozos estudiados hasta el momento en la cuenca del Colorado, es significativa su desaparición en depósitos no más jóvenes que Mioceno tardío, posiblemente reflejando cambios ambientales. La última presencia de esta especie se registra en sedimentos atribuidos al Mioceno tardío en los pozos Ombucta x-1 (Guerstein y Guler, 2000) y Cx-1 (Guerstein y Junciel, 2001) y al Mioceno medio de los pozos Fx-1 y Dx-1.

Género Lingulodinium Wall 1967 emend. Wall y Dale, en Wall et al. (1973)

Especie tipo: Lingulodinium machaerophorum (Deflandre y Cookson) Wall 1967.

\section{Lingulodinium hemicystum McMinn 1991 Fig. $7 \mathrm{j}-1$}

Dimensiones: Diámetro del cuerpo central, $30(45,7) 55 \mu \mathrm{m}$; largo de los procesos $4(7,6) 10 \mu \mathrm{m}$ (26 ejemplares medidos).

Observaciones: Se diferencia de L. machaerophorum por presentar arqueopilo epicistal y de L. brevispinosum Matsuoka y Bujak 1988 porque tiene los procesos largos y acuminados en lugar de redondeados distalmente. La mayoría de los ejemplares de Lingulodinium hallados en este estudio presentan arqueopilo hemicistal. Edwards (en Head, 1994b), señala la importancia de la diferenciación de los tipos de arqueopilo en los ejemplares de Lingulodinium, teniendo en cuenta que la presencia de arqueopilo hemicistal parece no ocurrir con anterioridad al Mioceno.

Paleoecología: Esta especie fue creada por McMinn (1991) en sedimentos provenientes de estuarios actuales de Australia, quien sugiere para esta especie condiciones de baja salinidad.

\section{Lingulodinium machaerophorum \\ (Deflandre y Cookson) Wall 1967 \\ Fig. $7 \mathrm{~g}-\mathrm{i}$}

Dimensiones: Diámetro del cuerpo central, 33,5 (46,5) 55 $\mu \mathrm{m}$; largo de los procesos, $6(11,2) 20 \mu \mathrm{m}$ (13 ejemplares medidos)

Observaciones: La superficie del cuerpo central está fuertemente granulada y cubierta por una capa de pelos muy finos y apretados, dando a la pared un aspecto muy denso y esponjoso.

Paleoecología: Es una especie eurihalina con distribución templada a tropical (Edwards y Andrle, 1992, y referencias allí citadas) y afinidad con ambientes estuarinos (Wall et al., 1977). En sedimentos modernos del Atlántico Norte, los porcentajes de L. machaerophorum son más altos cuando la temperatura del agua superficial en invierno está por encima de $\operatorname{los} 15^{\circ} \mathrm{C}$ y en verano supera $\operatorname{los} 27^{\circ} \mathrm{C}$ (Edwards y Andrle, 1992).

\section{Género Operculodinium (Wall) Matsuoka et al., 1997}

Especie tipo: Operculodinium centrocarpum (Deflandre y Cookson 1955) Wall 1967.

Operculodinium centrocarpum (Deflandre y Cookson) Wall 1967 (= Hystrichosphaeridium centrocarpum Deflandre y Cookson 1955)

Fig. 7 a - e

\section{$\mathrm{y}$ \\ Operculodinium israelianum (Rossignol 1961) Wall 1967}

Fig. $7 \mathrm{f}$

Descripción: Quistes esféricos, de tamaño variable. Pared esponjosa, superficie fibro-reticulada; procesos fibrosos particularmente en la base, de extremos usualmente expandidos o raramente acuminados. Algunos ejemplares presentan cierta alineación de los procesos en el área cingular.

Dimensiones: A partir del largo de los procesos y de la relación largo de los procesos/diámetro del cuerpo central, los ejemplares medidos se separaron en dos grupos: 1) Diámetro del cuerpo central $40(57,1) 80 \mu \mathrm{m}$; largo de los procesos, 9 (12) $17 \mu \mathrm{m}$; espesor de la pared, $1,1(1,5) 2 \mu \mathrm{m}$;

Figura 4. a, b Pentadinium laticinctum subsp. laticinctum Gerlach (x 600), UNSLP 0801t 27,5/107; a, superficie ventral, foco alto, ventral surface, high focus; $\mathbf{b}$, superficie dorsal, foco bajo, dorsal surface, low focus. c, f, i Hystrichosphaeropsis obscura Habib (x 650), UNSLP 4299 43,3/111,2; c, superficie ventral, foco bajo, ventral surface, low focus; $\mathbf{f}$, corte óptico, cross section; i, superficie dorsal, foco alto, dorsal surface, high focus. d, e Distatodinium paradoxum (Brosius) Eaton (x 650), UNSLP 5099 23,7/105,4, vista lateral, lateral view; d, foco alto, high focus; e, foco bajo, low focus. $\mathbf{g}$ - h, $\mathbf{j}$ - I Cousteaudinium aubryae de Verteuil y Norris, g, h (x 550), UNSLP 0501 42,5/97; g, corte óptico, cross section; $\mathbf{h}$, superficie apical, foco alto, apical surface, high focus; $\mathbf{j}$ - $\mathbf{l}$ (х 500), UNSLP 0601t, j, superficie apical, foco alto, apical surface, high focus; $\mathbf{k}$ corte óptico, cross section; $\mathbf{1}$, superficie antapical, foco bajo, antapical surface, low focus. Estos ejemplares muestran amplia separación entre endo y perifragma, these specimes show high degree of separation between endoblast and periblast. 

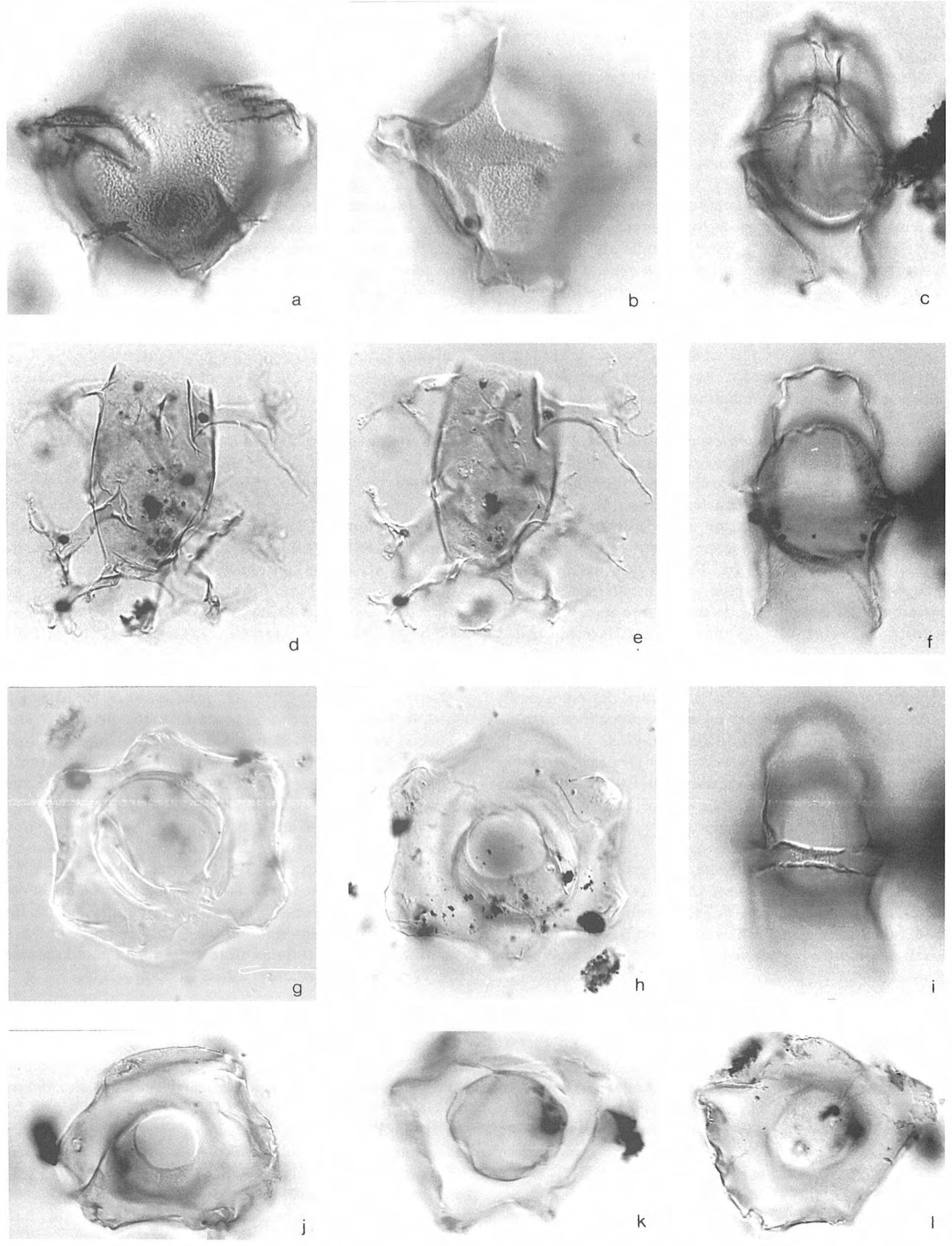
largo de los procesos/diámetro del cuerpo central, $0,15(0,2)$ 0,3 (32 ejemplares medidos) y 2) Diámetro del cuerpo central, 45 (60) $80 \mu \mathrm{m}$; largo de los procesos, 5 (7,7) $10 \mu \mathrm{m}$; espesor de la pared 1 (1,3) $2 \mu \mathrm{m}$; largo de los procesos/diámetro del cuerpo central, $0,09(0,12) 0,14$ (14 ejemplares medidos).

Observaciones: El largo de los procesos varía dentro de una misma muestra. Sin embargo, en las muestras donde estas especies son dominantes, se observa que la mayoría de los ejemplares tienen procesos entre 9 y $17 \mu \mathrm{m}$ y tienen una relación largo de los procesos/diámetro del cuerpo entre 0,15 y 0,3 . Este conjunto de ejemplares tienen procesos con extremos distales expandidos y son asignables a Operculodinium centrocarpum. Los

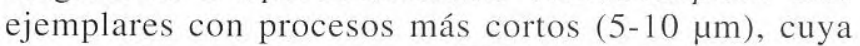
relación largo de los procesos/diámetro del cuerpo es de 0,09-0,14 son menos frecuentes y son asignables a Operculodinium israelianum, los extremos de los procesos pueden ser ocasionalmente acuminados.

Discusión: Operculodinium centrocarpum (sensu stricto) y $O$. israelianum tienen morfologías similares; $O$. centrocarpum tiene los procesos más largos aunque sus morfologías se superponen haciendo difícil su separación. Por este motivo, en vista de estas similitudes, Head (1998) agrupó estas dos especies en el complejo $O$. centrocarpum/israelianum. Pese a que los registros neógenos de $O$. centrocarpum y $O$. israelianum sugieren suficiente superposición de caracteres para poner en sinonimia estas dos especies, las distribuciones estratigráficas no son idénticas. Operculodinium israelianum se encuentra en sedimentos modernos y se registra frecuentemente en asociaciones del Neógeno y Cuaternario. O. centrocarpum es una especie extinguida y su distribución estratigráfica es dudosa debido a la confusión en la literatura con Operculodinium centrocarpum sensu Wall y Dale 1966. Esta última es una especie menos robusta y común en sedimentos modernos y cuaternarios del Atlántico Norte (Head, 1996).

Paleoecología: O. israelianum es común en ambientes actuales tropicales y templados cálidos (Edwards y Andrle, 1992), aunque también fue hallado en ambiente templado moderado. Esta especie habita profundidades someras, particularmente estuarios. Según Head (1996) la paleoecología de $O$. centrocarpum es desconocida. Los registros de Operculodinium centrocarpum en ambientes modernos corresponden a Operculodinium centrocarpum sensu Wall y Dale 1966.

\section{Operculodinium? sp. 1}

Fig. 8 a - g

\begin{abstract}
Descripción: Quiste pequeño escolocorado, cuerpo central esférico y elipsoidal. Pared compuesta por dos capas, una capa basal muy delgada $(<2 \mu \mathrm{m})$ y una capa externa más gruesa en cuya superficie se observa un microrretículo irregular con lúmenes menores de $5 \mu \mathrm{m}$. Procesos sólidos, no tabulares, circulares en sección transversal, ahusados a cónicos, a menudo curvados a sinuosos distalmente, con extremos acuminados y en algunos casos expandidos de forma capitada. Espaciamiento entre 2 y $5 \mu \mathrm{m}$, variable en un mismo ejemplar. Arqueopilo precingular pentagonal Tipo P (por pérdida de la placa 3") "deltacamerate" en el sentido de De Verteuil y Norris (1996).
\end{abstract}

Dimensiones: Diámetro máximo del cuerpo central, 25 $(32,7) 40 \mu \mathrm{m}$; largo de los procesos, 3 (4) $5 \mu \mathrm{m}$; espesor de la pared, $1(1,2) 1,8 \mu \mathrm{m}$ (25 ejemplares medidos).

Observaciones: Operculodinium? sp. 1 es asignado al género en forma dudosa porque a diferencia de la especie tipo, Operculodinium centrocarpum, los procesos de Operculodinium? sp. 1 son sólidos, acuminados y no fibrosos, así como la superficie es microrreticulada en lugar de fibrosa. O. ? erikianum (Head et al.) Head 1997 tiene los procesos más largos y crestas desarrolladas en la base de los procesos, no observadas en Operculodinium? sp. 1. O. janduchenei Head et al. 1989 c tiene la pared granulada y los procesos son huecos. O. placitum Drugg 1967 tiene los procesos más cortos y la superficie de la pared pilosa. Operculodinium? sp. 1 presenta marcada similitud con Operculodinium sp. 3 de De Verteuil y Norris (1996), siendo este último de mayor tamaño y con una pared alveolada y microgranulada. $O$. placitum y Operculodinium sp. 3 fueron descritos por De Verteuil y Norris (1996) con microscopio de barrido electrónico, pudiendo observarse claramente la superficie de las paredes. Si bien Operculodinium? sp. 1 es muy similar a ambas especies, no fue posible describir la pared con ese nivel de detalle, haciendo difícil su comparación.

Figura 5. a - c, Cribroperidinium tenuitabulatum (Gerlach) Helenes (x 550), UNSLP 0701 35,3/104,2; a, b, superficie ventral, ventral surface; a, foco bajo, low focus; $\mathbf{b}$, foco intermedio, intermediate focus; $\mathbf{c}$, superficie dorsal, foco alto, dorsal surface, high focus. d - f, Impagidinium sp. 2 (x 450), UNSLP 999 42,5/106; d, e, corte óptico, se observan las crestas perforadas, cross section showing perforate crests; d, superficie ventral, foco bajo, se observan las placas 1' y 4' separadas y el área sulcal, ventral surface, low focus, showing separated plates I' and 4 and the sulcal area. $\mathbf{g}$, $\mathbf{j}$ I, Impagidinium sp. 3 (x 750); g, UNSLP 4299 35,7/96,5, vista lateral, corte óptico, lateral view, cross section; j, k, UNSLP 4199 37/93; j, superficie ventral, foco bajo, ventral surface, low focus; $\mathbf{k}$, superficie dorsal, foco alto, dorsal surface, high focus; 1, UNSLP 1099 35,3/97, superficie antapical, foco bajo, antapical surface, low focus. h, i, Impagidinium sp. 1 (x 750), UNSLP 1099 33,5/103; h, superficie ventral, foco alto, ventral surface, high focus; i, superficie dorsal, foco bajo, dorsal surface, low focus. 

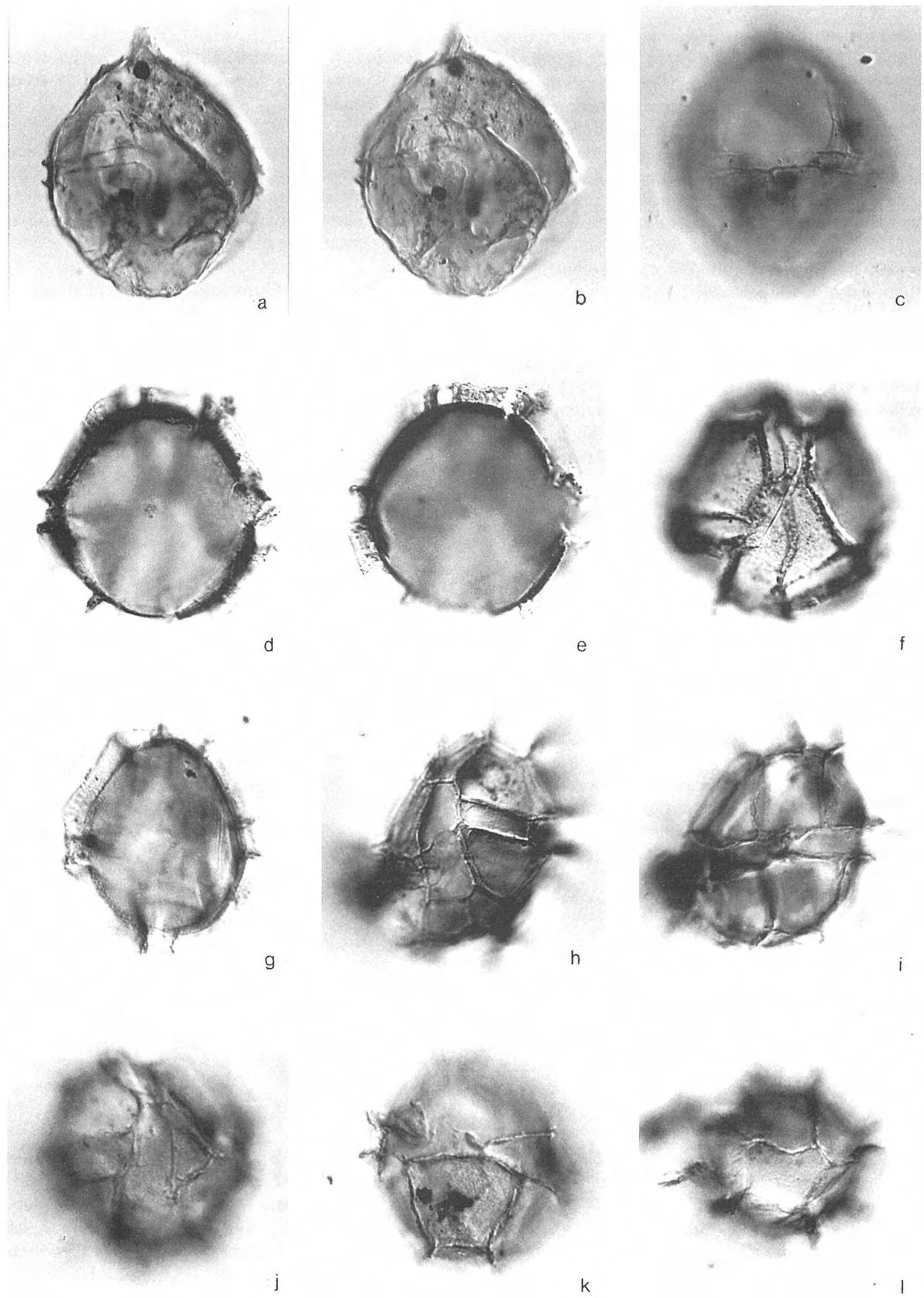


\section{Operculodinium? sp.2}

Fig. $8 \mathrm{~h}-\mathrm{n}$

\begin{abstract}
Descripción: Quiste pequeño, esférico y en la mayoría de los casos ovoidal. Superficie de la pared microgranulada. Procesos sólidos, baculados en sus bases, no tabulares y uniformemente distribuidos; cilíndricos y subcónicos, en algunos casos elongados, truncados o expandidos distalmente. Dentro de un mismo ejemplar pueden observarse uno o más de un tipo de procesos. Espaciamiento de los procesos entre 4 y $7 \mu \mathrm{m}$. Arqueopilo precingular, probablemente formado por la pérdida de la placa 3".
\end{abstract}

Dimensiones: Diámetro máximo del cuerpo central, 25 $(35,3) 40 \mu \mathrm{m}$; largo de los procesos $1,5(2,7) 5 \mu \mathrm{m}$, espesor de la pared $1(1,1) 1,5 \mu \mathrm{m}$ (11 ejemplares medidos).

Observaciones: Operculodinium? sp. 2 se diferencia de Operculodinium sp. 2 descrito en Head (1997) por la forma exclusivamente esférica y la homogeneidad en la forma de los procesos de esta última especie. De Verteuil y Norris (1996) consideran que la variabilidad morfológica de $O$. piaseckii Strauss y Lund 1992 puede ser agrupada en dos tipos básicos sobre la base de los procesos, sólidos subcónicos por un lado y cilíndricos y huecos por otro. Operculodinium? sp. 2 presenta similitud con los ejemplares que presentan procesos sólidos y subcónicos, aunque difiere por la forma variable de los procesos. Es muy similar a O. variabile Zevenboom y Santarelli 1995 en Zevenboon (1995) descrito para el Mioceno Medio de Italia. Si bien dicha especie presenta variabilidad en la forma de los procesos, tiene los procesos huecos. Operculodinium? sp. 2, al igual que Operculodinium? sp. 1, se asigna al género en forma dudosa debido a que, a diferencia de la especie tipo, $O$. centrocarpum, no tienen procesos fibrosos y la superficie del cuerpo central es microgranulada en lugar de fibro-reticulada.

\section{Operculodinium sp. 3}

Fig. 8 o-p

Descripción: Quiste esférico a elipsoidal (probablemente por deformación). Procesos muy finos, densa y regularmente distribuidos, aciculares, con los extremos distales acuminados y en algunos casos capitados. Arqueopilo observado en pocos ejemplares, generalmente el opérculo está "in situ", probablemente constituido por la placa 3". Pared cribosa, microrreticulada entre las espinas, entre 1 y $1,5 \mu \mathrm{m}$ de espesor.

Dimensiones: Diámetro máximo del cuerpo central, 39 $(43,1) 52 \mu \mathrm{m}$; largo de los procesos, $6(7,9) 10 \mu \mathrm{m}(13$ ejemplares medidos).

Observaciones: Operculodinium sp. 3 es semejante a $O$. centrocarpum (Deflandre and Cookson) Wall 1967, difiere principalmente en el tipo y distribución de los procesos. Estos son más delgados, sinuosos, con aspecto piloso y densamente distribuidos.

Subfamilia Gonyaulacoideae Autonimia Género Bitectatodinium Wilson 1973

Especie tipo: Bitectatodinium tepikiense Wilson 1973.

\section{Bitectatodinium tepikiense Wilson 1973 Fig. 9 e, f}

Dimensiones: Diámetro máximo $38(38,7) 40 \mu \mathrm{m}(3$ ejemplares medidos).

Paleoecología: El material tipo del Pleistoceno medio de Nueva Zelanda está asociado a un clima templado a cálido. Sin embargo, registros de B. tepikiense en sedimentos actuales del Atlántico Norte muestran una distribución templada a templada fría (Head, 1996 y referencias ahí citadas).

Distribución estratigráfica: El registro más bajo de Bitectatodinium tepikiense corresponde al Mioceno superior de Nueva Jersey y del Mar de Noruega (Rochon et al., 1999 y referencias allí citadas).

Género Unipontidinium Wrenn 1988

Especie tipo: Unipontidinium aquaeductum (Piaseckii 1980) Wrenn 1988.

\section{Unipontidinium sp.} Fig. $10 \mathrm{a}-\mathrm{c}$

Descripción: Quiste corado, cuerpo central subesférico, procesos trifurcados con extremos distales expandidos y

Figura 6. a - c Dapsillidinium pseudocolligerum (Stover) Bujak et al. (x 850), UNSLP 4199 47/102,5; a, foco alto, high focus; $\mathbf{b}$, foco intermedio, intermediate focus; $\mathbf{c}$, corte óptico, cross section. $\mathbf{d}-\mathbf{f}$ Labrynthodinium truncatum subsp. truncatum Piasecki (x 1000), UNSLP 1299 45/102,5; d, vista apical oblicua, foco alto, oblique apical view, high focus; e, f, (ca. 900) UNLP 5199 33,5/99; e, corte óptico, cross section; f, foco bajo, low focus. g, h Reticulatosphaera actinocoronata Matsuoka (x 950), UNSLP 2199 35/110,4; g, corte óptico, cross section; h, vista general, general view. i - I Hytrichokolpoma rigaudiae Deflandre y Cookson (x 650); i, l, UNSLP 5099 44,5/108,3; i, superficie dorsal, foco alto, dorsal surface, high focus; $\mathbf{1}$, corte óptico, cross section; j, k, UNSLP 4299 43,3/111,2; j, superficie ventral, foco intermedio, se observa la depresión sulcal, ventral surface, intermediate focus showing the sulcal notch; $\mathbf{k}$, superficie dorsal, foco bajo, dorsal surface, low focus. 

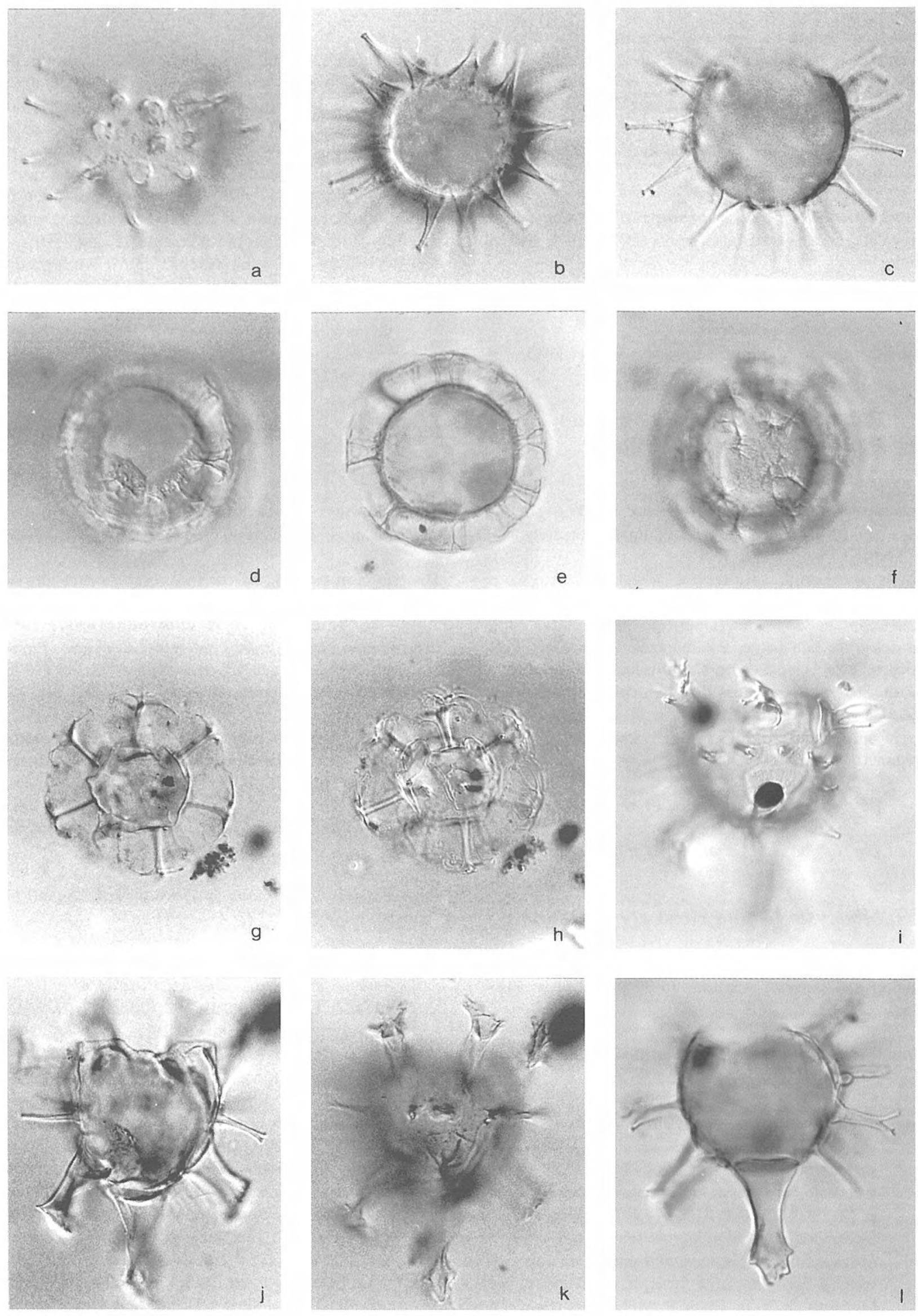
conectados por una trabécula simple. Los procesos son membranosos, con un eje central sólido y algunos ejemplares presentan una cavidad proximal. Una delgada membrana cubre las trabéculas a modo de cinta alrededor de toda la red trabecular.

Dimensiones: Diámetro máximo del cuerpo central, $32 \mu \mathrm{m}$; diámetro máximo total, $63 \mu \mathrm{m}$; largo de los procesos, entre 13 y $18 \mu \mathrm{m}$.

Observaciones: El único ejemplar hallado no es comparable con ninguna especie descrita hasta el momento.

\section{Género Cannosphaeropsis Wetzel 1933}

Especie tipo: Cannosphaeropsis utinensis Wetzel 1933.

\section{Cannosphaeropsis quattrocchiae Guerstein et al. 2001}

Fig. $11 \mathrm{a}-\mathrm{d}$

Dimensiones: Diámetro de cuerpo central $35(39,6) 45 \mu \mathrm{m}$; diámetro total $55(74,8) 108 \mu \mathrm{m}$ (23 ejemplares medidos).

Registros previos: C. quattrocchiae está descrito en la cuenca del Colorado en sedimentos de probable edad Miocena temprana/Oligocena (Guerstein et al., 2001). En la cuenca del golfo San Jorge, Palamarczuk y Barreda (1998) identificaron esta especie como Cannosphaeropsis utinensis, en sedimentos provenientes de la Formación Chenque asignados al Mioceno temprano. Hasta el momento ha sido registrada únicamente en el Cenozoico medio del Atlántico suroccidental.

\section{Género Habibacysta Head et al. 1989b}

Especie tipo: Habibacysta tectata Head et al. 1989b.

\section{Habibacysta tectata Head et al. 1989 b Fig. 9 j -1}

Dimensiones: Diámetro máximo, 35-37 $\mu \mathrm{m}$ (2 ejemplares medidos).

Distribución estratigráfica: Mioceno Medio a Pleistoceno, posiblemente hasta el Pleistoceno superior bajo (Head et al. 1989b, Head, 1994a y referencias allí citadas).

Paleoecología: Según Head (1994a) la adaptación de esta especie a ambientes neríticos internos está indicada por la presencia en depósitos correspondientes a profundidades entre 40 y $8 \mathrm{~m}$ o menores. El transporte hacia profundidades mayores y la redepositación explican posiblemente su presencia en ambientes neríticos externos y oceánicos en el Neógeno del Atlántico Norte; tolera una gama amplia de factores ecológicos, aunque en su mayoría están asociados a condiciones frías. Head (1996) indica que esta especie está restringida a los intervalos más fríos en el Plioceno del Mediterráneo.

Género Hystrichosphaeropsis Deflandre 1935

Especie tipo: Hystrichosphaeropsis ovum Deflandre 1935.

\section{Hystrichosphaeropsis obscura Habib 1972 Fig. 4 c, f, i}

Dimensiones: Largo total, $60(82,8) 101 \mu \mathrm{m}$; largo del cuerpo central, $40(49,6) 60 \mu \mathrm{m}$ (19 ejemplares medidos).

Distribución estratigráfica: La presencia más alta de esta especie, en el Mioceno superior alto, define el techo de la Zona de Intervalo Hystrichosphaeropsis obscura determinada para el talud continental de Nueva Jersey (De Verteuil, 1996). De acuerdo con Williams et al. (1998b), el biocrón se extiende desde el Rupeliense (30,7 Ma) hasta el Tortoniense (7,3 Ma).

En la cuenca del Colorado ha sido hallada en sedimentos de posible edad serravalianiense-tortoniense o más antigua (Guerstein y Guler, 2000; Guerstein y Junciel, 2001).

Género Pentadinium Gerlach 1961 emend. Benedek et al. 1982

Especie tipo: Pentadinium laticinctum Gerlach 1961 emend. Benedek et al. 1982.

\section{Pentadinium laticinctum}

Gerlach 1961 emend. Benedek et al. 1982

Fig. $4 \mathrm{a}-\mathrm{b}$

Figura 7. a-e, Operculodinium centrocarpum (Deflandre y Cookson) Wall; a, (x 450) UNSLP 5199 26/97, superficie ventral, foco alto, ventral surface, high focus; b, (x 600) UNSLP 1499 25/96, superficie dorsal, foco bajo, dorsal surface, low focus; c, (x 1000) UNSLP 1899 30,5/99, opérculo libre, operculum free; d, (x 500), superficie ventral, foco bajo, se observa la estructura fibrosa de la pared, ventral surface, low focus, showing the fibrous wall structure; e, (x 750) UNSLP 2199 44,6/105, superficie dorsal, foco intermedio, dorsal surface, intermediate focus. f, Operculodinium israelianum (Rossignol) Wall; (x 650), UNSLP 999 24,5/101,5, corte óptico, cross section. g - i Lingulodinium machaerophorum (Deflandre y Cookson) Wall (x 750) UNSLP 1499 41/99; vista lateral, lateral view; $\mathbf{g}$, foco alto, high focus; $\mathbf{h}$, corte óptico, cross section; $\mathbf{i}$, foco bajo, low focus. $\mathbf{j}-\mathbf{l}$, Lingulodinium hemicystum McMinn (x 700), UNSLP 24/93; superficie apical, apical surface; j, foco alto, high focus; k, foco intermedio, intermediate focus; $\mathbf{1}$, corte óptico, cross section. 

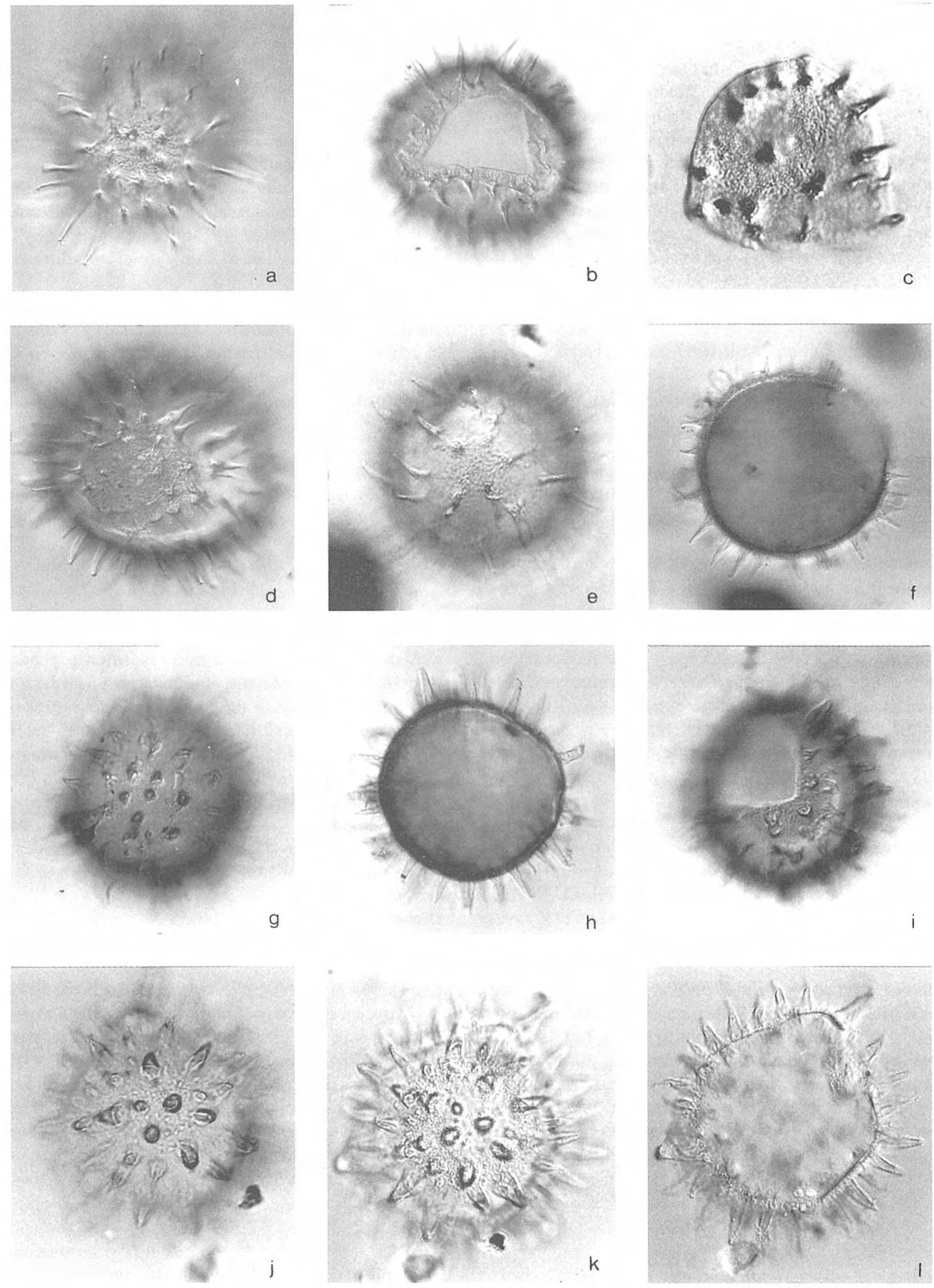
Dimensiones: Diámetro máximo 50 (75) $99 \mu \mathrm{m}(14$ ejemplares medidos).

Registros previos: Según Head et al. (1989c) la distribución de P. laticinctum se extiende desde el Eoceno medio hasta el Mioceno tardío. Powell (1992) registra la última presencia de esta especie en la Biozona de Intervalo Heteraulacysta verricula (Hve) que se extiende desde el Serravaliense (parte) al Tortoniense? (parte). De acuerdo con Williams et al. (1998b), la última aparición confirmada de P. laticinctum corresponde al límite Serravaliense/Tortoniense a los 11,2 Ma. En Argentina fue hallada en sedimentos asignados al Mioceno tardío o más antiguos provenientes de las perforaciones Ombucta $\mathrm{x}-1$ y Cx-1; Dx-1 y Fx-1 en la Cuenca del Colorado (Guerstein y Guler, 2000; Guerstein y Junciel, 2001).

\section{Género Tectatodinium Wall 1967 emend. Head 1994}

Especie tipo: Tectatodinum pellitum Wall 1967 emend. Head 1994.

\section{Tectatodinium pellitum Wall 1967 emend. Head 1994 \\ Fig. 9 g - i}

Dimensiones: Diámetro máximo, $40(49,1) 55 \mu \mathrm{m}$; espesor de la pared, $3(3,9) 5 \mu \mathrm{m}$ (19 ejemplares medidos).

Observaciones: La pared esponjosa y el arqueopilo con márgenes irregulares distinguen a Tectatodinium pellitum de Habibacysta tectata, cuya pared es columelada/tegilada. En Bitectatodinium tepikiense la pared consiste en una disposición compleja de proyecciones muy finas, perpendiculares o subperpendiculares a una capa basal más delgada.

Distribución estratigráfica: $T$. pellitum es uno de los quistes producido por el complejo Gonyaulax spinifera (Claparède y Lachmann 1859) Diesing 1866 (Head, 1994a). La primera aparición de esta especie se registra en el Eoceno inferior (Head, 1994a y referencias citadas allí).

Paleoecología: Tectatodinium pellitum se distribuye en áreas templadas a subtropicales en el Atlántico Norte (Harland, 1983). Raramente constituye más del 10\% de las asociaciones de quistes de dinoflagelados y la abundancia máxima ocurre cuando la temperatura del agua de superficie en verano varía entre 15 y $21^{\circ} \mathrm{C}$ y en invierno entre 8 y $10^{\circ} \mathrm{C}$ (Edwards y Andrle, 1992). Tiene una distribución nerítica a oceánica y ha sido registrado en sedimentos estuarinos (Wall et al., 1977).
Género Impagidinium Stover y Evitt 1978

Especie tipo: Impagidinium dispertitum (Cookson y Eisenack) Stover y Evitt 1978.

\section{Impagidinium sp. 1}

Fig. 5 h, i

Descripción: Quiste subesférico de pared lisa, ornamentado con crestas parasuturales que definen la paratabulación. En las regiones sulcal y apical la paratabulación está incompletamente delineada. El arqueopilo está formado por la pérdida de la placa 3"'. Tabulación inferida: 4', 6", 6c, 6",', 1p, 1ps, 1,",".

Comparaciones: El ejemplar hallado presenta marcada similitud con I. californiense Damassa 1979 descrito para el Mioceno de California. Sin embargo, en el único ejemplar hallado, no se observan el área apical y el arqueopilo que son características diagnósticas de la especie.

Dimensiones: Diámetro máximo del cuerpo, $44 \mu \mathrm{m}$; largo de las crestas, $4 \mu \mathrm{m}$ ( 1 ejemplar medido).

\section{Impagidinium sp. 2}

Fig. $5 \mathrm{~d}-\mathrm{f}$

Descripción: Quiste esférico a subesférico. Crestas parasuturales estriadas transversalmente y perforadas principalmente de forma elongada en la dirección de las estrías. Pared gruesa de hasta $3 \mu \mathrm{m}$ de espesor, con la superficie rugosa. Cíngulo fuertemente helicoidal, paratabulación del sulco incompleta, apenas se observa el comienzo de algunas crestas y el margen posterior de la placa sulcal anterior. La cresta que separa las placas 1' y 4 ' está desarrollada y los márgenes laterales son paralelos, dándole a las placas una forma rectangular. La placa 6" es de forma subtriangular a trapezoidal.

Dimensiones: Diámetro máximo del cuerpo, 60 (67) $74 \mu \mathrm{m}$; crestas entre 6 y $10 \mu \mathrm{m}$ ( 3 ejemplares medidos).

Comparaciones: Impagidinium sp. 2 difiere del resto de las especies de Impagidinium descritas hasta el momento. Se asemeja a $I$. brevisulcatum en el área apical, particularmente en la forma de las placas 1' y 4', cuyos márgenes laterales son paralelos, y en la forma trapezoidal de la placa 6". I. fenestroseptatum Head et al. (1989b) descrito para el Plioceno Temprano del Mar del Labrador, presenta perforaciones de mayor tamaño que se desarrollan en la mitad distal de las crestas.

Figura 8. a - g Operculodinium? sp. 1 (x 1000); a, b, UNSLP 4999 40/105,9; superficie ventral, ventral surface; a, foco alto, high focus; b, foco intermedio, intermediate focus; c, g, UNSLP 1699 41,4/104,9, superficie ventral, ventral surface; c, foco alto, high focus; g, foco intermedio, intermediate focus; d, UNSLP 1599 36/98, superficie dorsal, foco bajo, óperculo "in situ", dorsal surface, low focus, operculum in place; e, f, UNSLP 1999 47/104,5, vista 

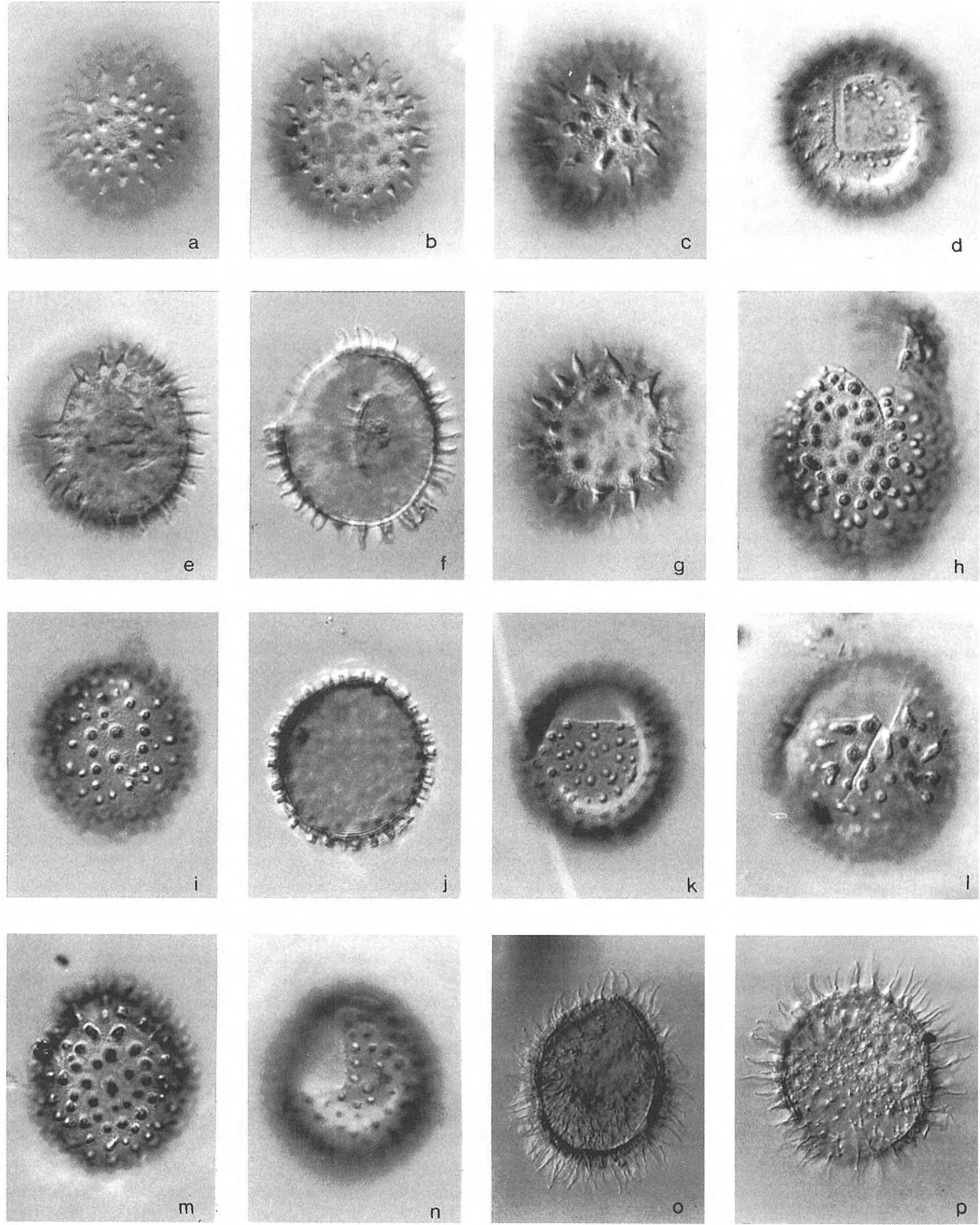

lateral, lateral view; e, foco intermedio, intermediate focus; $\mathbf{f}$, corte óptico, cross section. $\mathbf{h}$ - $\mathbf{n}$ Operculodinium? sp. 2 (x 1000); h, UNSLP 999 26/108,5, vista lateral, foco intermedio, lateral view, intermediate focus; i - k, UNSLP 999 21,4/105,5; i, superficie ventral, foco alto, ventral surface, high focus; j, corte óptico, cross section; k, superficie dorsal, foco bajo, dorsal surface, low focus; I, UNSLP 999 23/95,5, superficie dorsal oblicua, foco alto, oblique dorsal surface, high focus; m, n, UNSLP 1299 41,4/110,7; vista lateral, lateral view; m, foco alto, high focus; $\mathbf{n}$, foco bajo, low focus. o, p Operculodinium sp. 3 (x 650) UNSLP; o, 2399 24,5/99; corte óptico, cross section; p, UNSLP 2399 44/106, foco alto, high focus. 


\section{Impagidinium sp. 3}

Fig. 5 g, j - 1

Descripción: Quiste proximocorado, de tamaño mediano a pequeño y forma oval. Pared del cuerpo central hasta $2 \mu \mathrm{m}$ de espesor, superficie finamente granulada. Crestas parasuturales delgadas y transparentes definen la paratabulación. La paratabulación del sulco está poco definida, la cresta entre las placas 1' y 4' está parcial a completamente definida, la placa 6" es triangular y el arqueopilo está formado por la pérdida de la placa 3".

Dimensiones: Diámetro máximo del cuerpo central, 33 $(37,8) 50 \mu \mathrm{m}$; ancho de la cresta entre 2 y $5 \mu \mathrm{m}$ (7 ejemplares medidos).

Comparaciones: Impagidinium sp. 3 difiere de $I$. dispertitum (Cookson y Eisenack 1965) Stover y Evitt 1978 en que éste tiene mayores dimensiones $(55-80 \mu \mathrm{m})$ y el área sulcal está típicamente señalada por tres placas grandes. I. patulum (Wall 1967) Stover y Evitt 1978 presenta la paratabulación reducida en la superficie ventral y una placa compuesta representa a 1' + 4'. I. victorianum (Cookson y Eisenack 1965) Stover y Evitt 1978 es muy similar, si bien presenta dimensiones bastante mayores (80-120 $\mu \mathrm{m})$, la forma es esférica y está ausente la divisoria entre las placas 1' y 4'. I. maculatum (Cookson y Eisenack 1961) Stover y Evitt 1978 se asemeja a Impagidiniun sp. 3 pero sus dimensiones son mayores (52-64 $\mu \mathrm{m})$ y la superficie de la pared es moteada.

Género Nematosphaeropsis Deflandre and Cookson 1955 emend. Wrenn 1988

\section{Nematosphaeropsis rigida Wrenn 1988}

Fig. $10 \mathrm{~d}-\mathrm{m}$

Dimensiones: Largo total, $50(58,7) 72 \mu \mathrm{m}$; largo del cuerpo central, $30(35,9) 43 \mu \mathrm{m}$ (14 ejemplares medidos). Ejemplares con plataforma compleja: largo total, $50(60,7)$ $75 \mu \mathrm{m}$; largo del cuerpo central, 27 (35) $46 \mu \mathrm{m}$ (19 ejemplares medidos).

Observaciones: Los ejemplares hallados muestran variabilidad con respecto a las uniones gonales en los extremos distales de los procesos y a la doble trabécula que une los procesos. Si bien todos los ejemplares estudiados se ajustan en rasgos generales a Nematosphaeropsis rigida, es posible diferenciar dos características morfológicas no descritas previamente. Por un lado, se observaron ejemplares cuyas trabéculas presentan sinuosidades y entrecruzamientos en algunos sectores de la red externa trabecular (Fig. 10 j - 1). Los especimenes ilustrados en Wrenn (1988, lám. 4, figs. 15) muestran esta característica aunque no fue descrita en el momento en que esta especie fue creada. Otros ejemplares se caracterizan por el desarrollo de plataformas perforadas en los extremos distales de los procesos, observadas en un número importante de ejemplares (Fig. 10 f, m). Este último rasgo justificaría la creación de una subespecie de Nematosphaeropsis rigida (Wrenn com. pers.). Sin embargo, los ejemplares no se encuentran suficientemente bien conservados.

\section{Género Spiniferites Mantell 1850, emend Sarjeant 1970}

Especie tipo: Spiniferites ramosus (Ehrenberg 1838) Loeblich y Loeblich 1966.

\section{Spiniferites falcipedius Warny y Wrenn 1997}

Fig. 3 a - c

Dimensiones: Largo total, 65 (75) $85 \mu \mathrm{m}$; largo del cuerpo central, $40(52,5) 65 \mu \mathrm{m}$; largo de los procesos, 14 (17) 20 $\mu \mathrm{m}$; ancho de la estructura de los procesos antapicales, 20 (22,5) $23 \mu \mathrm{m}$ (2 ejemplares medidos).

Observaciones: Spiniferites falcipedius se diferencia de $S$. mirabilis por tener la estructura antapical más sólida formada por dos procesos anchos, unidos por una membrana suturocavada. Además, S. mirabilis tiene procesos gonales e intergonales mientras que $S$. falcipedius no presenta procesos intergonales.

Distribución estratigráfica: Mioceno a Plioceno (Warny y Wrenn, 1997).

\section{Spiniferites cf. falcipedius Warny y Wrenn 1996 Fig. 3 g, h, k, 1}

Descripción: Quiste escolocorado, cuerpo central esférico a levemente elongado, procesos trifucados delgados. Los procesos son huecos, subtriangulares a circulares en sección transversal y conectados proximalmente por crestas bajas. Son generalmente gonales, aunque en algunos casos hay presencia de gonales e intergonales. Dos procesos antapicales anchos están unidos por una membrana suturocavada formando una estructura antapical resistente. El perifragma es microgranular.

Dimensiones: Largo total, 39 (48,6) $55 \mu \mathrm{m}$; largo del cuerpo central, 27 (34) $40 \mu \mathrm{m}$; largo de los procesos, 7 (10,2) 13 $\mu \mathrm{m}$; ancho de la estructura antapical $7(8,2) 9) \mu \mathrm{m}(12$ ejemplares medidos).

Observaciones: Los ejemplares muestran el tipo de estructura antapical típica de S. falcipedius, sin embargo, las dimensiones son menores, los procesos son más delgados, trifurcados y no presenta las terminaciones distales membranosas como en el caso de S. falcipedius. Se observaron ejemplares que comparten características de $S$. falcipedius y $S$. mirabilis; presentan dos procesos antapicales anchos típicos de $S$. falcipedius y procesos gonales e intergonales finos que es un rasgo de $S$. mirabilis. Esta gradación fue observada por Warny y Wrenn (1997) en las asociaciones de la cuenca Mediterránea, y sugieren la 


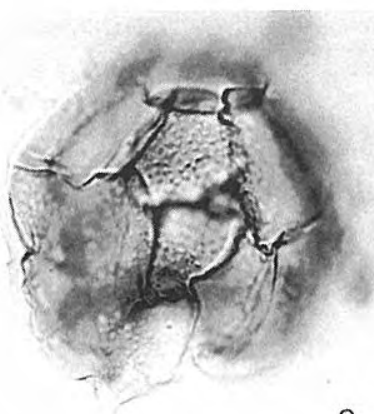

a
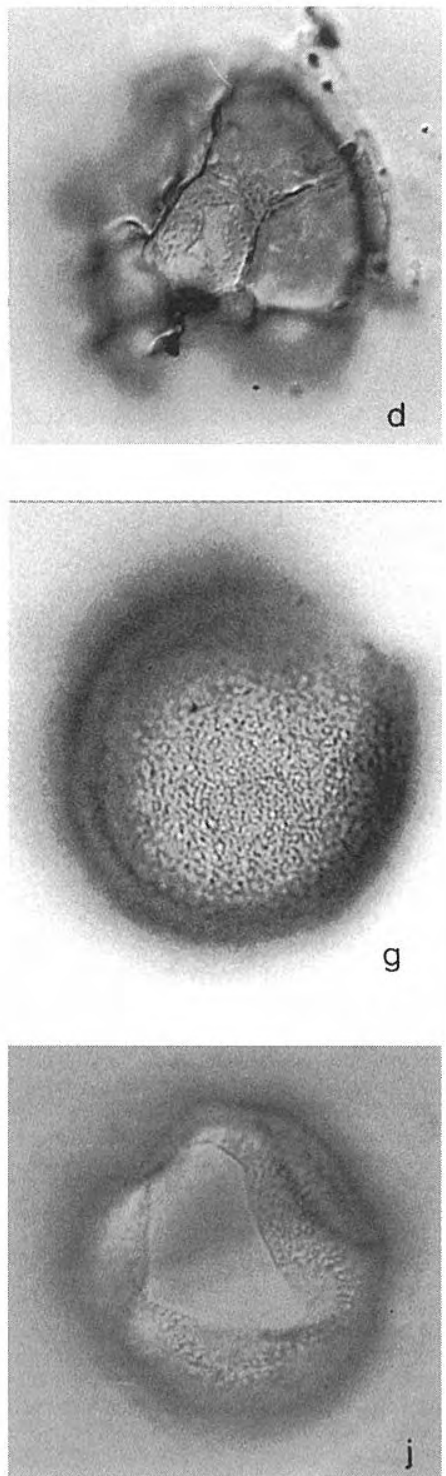
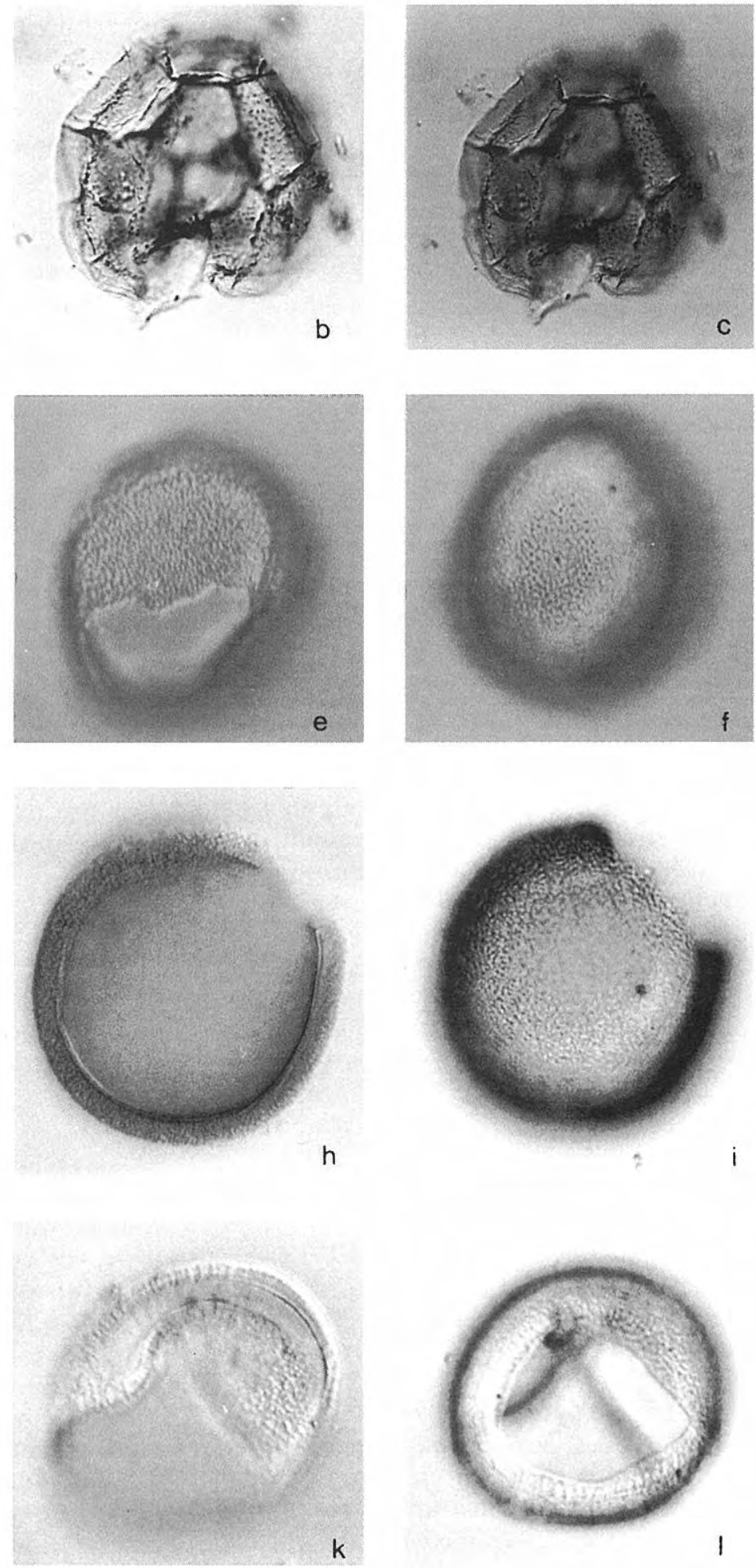

Figura 9. a - d, Heteraulacacysta sp. UNSLP 0701 32,5/108; a, b, (x 700) superficie apical, apical surface; a, foco alto, high focus; b, foco intermedio, intermediate focus; c, (x 600), superficie apical, foco intermedio, apical surface, intermediate focus; d, (x 700), superficie antapical, foco bajo, antapical surface, low focus. e - f, Bitectatodinium tepikiense Wilson (x 750), UNSLP 4299 43/110,5; e, superficie dorsal oblicua, foco alto, se observa el arqueopilo $2 \mathrm{P}$, oblique dorsal surface, high focus, showing type $2 \mathrm{P}$ arquepyle; $\mathbf{f}$, superficie ventral oblicua, foco bajo, oblique ventral surface, low focus. g- i, Tectatodinium pellitum (Wall) Head (x 850), UNSLP 5299 43/99, vista lateral, lateral view; $\mathbf{g}$, foco alto, high focus; $\mathbf{h}$, corte óptico, cross section; $\mathbf{i}$, foco bajo, low focus. $\mathbf{j}-\mathbf{l}$, Habibacysta tectata Head et al. (x 1000), j, UNSLP 1699 19/103,2, superficie dorsal, foco alto, dorsal surface, high focus; k, l, UNLP 1499 22,5/105; k, corte óptico, se observa la pared columelada, cross section, showing the collumelate wall structure; $\mathbf{I}$, superficie dorsal, foco bajo, dorsal surface, low focus. 
posible existencia de una relación evolutiva entre ambas especies.

\section{Spiniferites mirabilis (Rossignol 1964) Sarjeant 1970}

Fig. 3 d

Dimensiones: Largo del cuerpo, $40(46,5) 50 \mu \mathrm{m}$; largo de los procesos, 12 (13,5) $15 \mu \mathrm{m}$ (6 ejemplares medidos).

Observaciones: Wall et al. (1977) indican para esta especie una distribución nerítica y cosmopolita, asociada principalmente a la de Operculodinium centrocarpum. $\mathrm{La}$ distribución actual es nerítica interna a oceánica con afinidad a temperaturas cálidas (Harland, 1983) aunque de acuerdo con Edwards y Andrle (1992) su rango podría extenderse desde frío moderado a tropical.

Registros previos: Aunque hay algunos registros en el Paleoceno, esta especie es registrada con frecuencia en depósitos neógenos y cuaternarios (Head, 1996).

\section{Spiniferites / Achomosphaera spp.}

Observaciones: Estas especies son elementos mayoritarios en casi todas las asociaciones estudiadas, presentan alta diversidad morfológica con superposición de caracteres, haciendo difícil la asignación de las mismas. Por lo tanto, por razones prácticas y considerando la escasa información paleoecológica y bioestratigráfica brindada por estos ejemplares, se agruparon en Spiniferites / Achomosphaera spp. De todas maneras, ciertas especies, como Spiniferites mirabilis, S. falcipedius y Spiniferites cf. falcipedius, por implicancias paleoecológicas y/o por distinciones morfológicas, son estudiadas separadamente.

\section{Subfamilia Incierta}

\section{Género Melitasphaeridium Harland y Hill 1979}

Especie tipo: Melitasphaeridium choanophorum (Deflandre y Cookson 1955) Harland y Hill 1979.

Observaciones: Harland y Hill (1979) enfatizaron la extrema variabilidad de las terminaciones distales de los procesos en la descripción de la especie tipo. Strauss y Lund (1992) reconocieron e ilustraron una amplia variabilidad morfológica haciendo referencia al complejo Melitasphaeridium choanophorum en el Mioceno de Alemania. Estos autores consideraron los distintos tipos morfológicos como ecofenotipos, variedades o subespecies de $M$. choanophorum. La mayoría de los ejemplares hallados son asignables a $M$. choanophorum en el sentido de Harland y Hill (1979). Algunos de ellos presentan distintos tipos de procesos en un mismo ejemplar, correspondientes tanto a $M$. choanophorum como a $M$. choanophorum var. reductum, $M$. choanophorum var. B de Strauss y Lund 1992 y a formas transicionales a $M$. pseudorecurvatum.

\section{Melitasphaeridium chonaophorum (Deflandre y Cookson 1955) Harland y Hill 1979 \\ Fig. $11 \mathrm{j}-\mathrm{k}$}

Dimensiones: Diámetro del cuerpo central, 25 (33,5) $40 \mu \mathrm{m}$; largo de los procesos, 6 (11,3) $15 \mu \mathrm{m}$ (24 ejemplares medidos).

Observaciones: Esta especie presenta los extremos distales de los procesos expandidos en forma de embudo, bordeado por una serie de espinas cortas también variables en longitud.

\section{Melitasphaeridium choanophorum var. B de Strauss y Lund 1992 Fig. $11 \mathrm{e}$}

Dimensiones: Diámetro del cuerpo central, 30 (34,2) $37 \mu \mathrm{m}$; largo de los procesos $6(11,7) 15 \mu \mathrm{m}$ ( 5 ejemplares medidos).

Observaciones: Estos ejemplares exhiben procesos con márgenes distales enteros. Según Strauss y Lund (1992) y de acuerdo con Bujak et al. (1980), éste podría ser el estadío evolutivo más joven del complejo $M$. choanophorum.

\section{Melitasphaeridium? spp.}

Fig. $11 \mathrm{f}-\mathrm{i}, 1$

Dimensiones: Diámetro del cuerpo central, 25 (33,1) $40 \mu \mathrm{m}$; largo de los procesos, 8 (11) $16 \mu \mathrm{m}$ (14 ejemplares medidos).

Observaciones: Se incluyen aquí ejemplares con más de un proceso por placa con tendencia a una distribución no tabular. En algunos casos los ejemplares presentan procesos con extremos distales aserrados, de largo reducido y comparables con formas transicionales a Melitasphaeridium (?) cf. pseudorecurvatum (Morgenroth) Bujak et al. (1980). En otros, los ejemplares exhiben también mayor número de procesos, los extremos distales están levemente expandidos y en algunos casos presentan sólo una espina distal. En este último caso los ejemplares son similares a "Melitasphaeridium" sp. de Strauss y Lund (1992) quienes consideraron estas formas transicionales a Operculodinium. Sin embargo, los ejemplares asignados a Melitasphaeridium? spp. presentan una pared lisa, completamente diferente a la pared fibro-reticulada de Operculodinium.

Familia Goniodomaceae Lindemann 1928

Subfamilia Helgolandinioideae Fensome et al. 1993 Género Tuberculodinium Wall 1967

Especie tipo: Tuberculodinium vancampoae (Rossignol 1962) Wall 1967. 

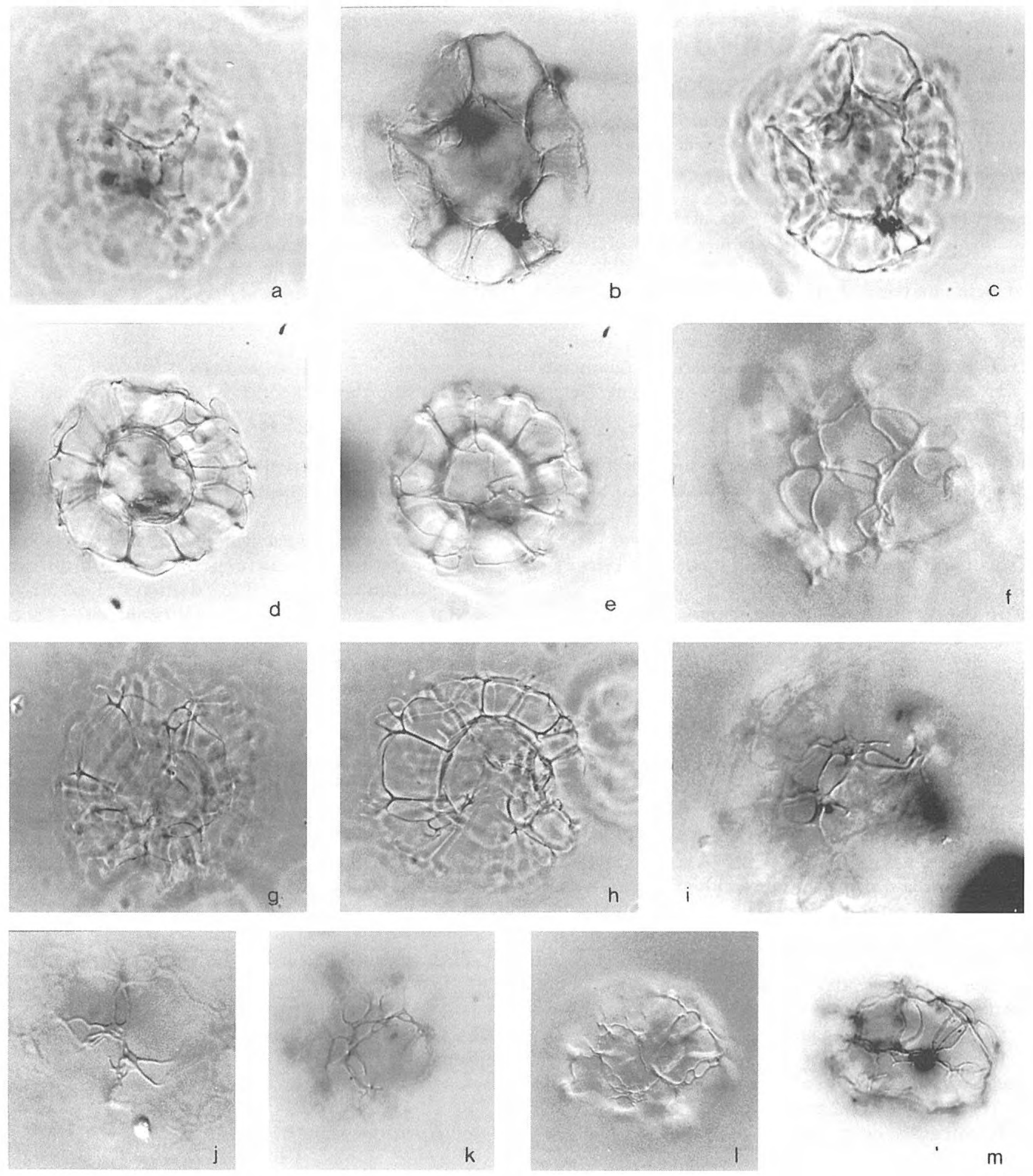

Figura 10. a - c, Unipontidinium sp. (x 600) UNSLP 999 33,5/103,5; a, foco alto, mostrando detalle de la trabécula membranosa, high focus showing membranous trabecula; b, corte óptico, mostrando los procesos membranosos, cross section showing membranous processes; c, vista general, general view. $\mathbf{d}-\mathbf{m}$, Nemmatosphaeropsis rigida Wrenn; $\mathbf{d}$, e, (x 500), UNSLP 4199 25/101,8; d, superficie ventral, foco intermedio, se observan trabéculas adicionales conectando procesos adyacentes, ventral surface, intermediate focus, showing aditional trabeculae joining adjacent processes; e, superficie dorsal, foco bajo, dorsal surface, low focus; $\mathbf{f}, \mathbf{m}$, detalle de la unión entre procesos adyacentes con triple trabécula, detail of triple trabeculae joining adjacent processes; f, (x 1000) UNSLP 4199 20,7/101; m (x 600) UNSLP 1999 43,5/104,9; g, h, (x 500) UNSLP 2499 41/95, vista general, general view; g, foco alto, high focus; h, foco intermedio, intermediate focus; i (x 1000) UNSLP 1399 23,5/94,5, detalle de plataformas gonales perforadas, detail of perforate gonal platforms; $\mathbf{j}, \mathbf{k}, \mathbf{l}$, detalle de plataformas gonales perforadas y uniones complejas entre procesos adyacentes, detail of perforate gonal platforms and complex trabecula joining adjacent processes; j, (x 1000) UNSLP 4299 45,5/100; k, (x 800), UNSLP 4199 25/101,8; l, (x 800), UNSLP 1899 31/99,5. 


\section{Tuberculodinium vancampoae \\ (Rossignol 1962) Wall 1967 \\ Fig. $3 \mathrm{~m}$}

Dimensiones: Diámetro del cuerpo central, $70(81,5) 93 \mu \mathrm{m}$; diámetro total, $100(114,5) 135 \mu \mathrm{m}$; largo de los tubérculos, 12 (16) $20 \mu \mathrm{m}$ (7 ejemplares medidos).

Paleoecología: Esta especie está documentada en ambientes neríticos internos a externos, con preferencia en climas tropical a subtropical y templado cálido. Las abundancias mayores del $2 \%$ se registraron solamente donde la temperatura del agua superficial supera $\operatorname{los} 27^{\circ} \mathrm{C}$ en verano y $\operatorname{los} 16^{\circ} \mathrm{C}$ en invierno (Edwards y Andrle, 1992). Actualmente está asociada a estuarios y raramente está presente en profundidades mayores que $60 \mathrm{~m}$ (Head, 1996 y referencias allí citadas).

Distribución estratigráfica: Oligoceno superior a Actual (Head y Westphal, 1999 y referencias allí citadas).

Subfamilia Goniodomoideae (Autonimia) Género Heteraulacacysta Drugg y Loeblich 1967

Especie tipo: Heteraulacacysta campanula Drugg y Loeblich 1967.

\section{Heteraulacacysta sp. 1}

Fig. 9 a - d

Descripción: Quiste discoidal con compresión polar, aproximadamente circular en vista polar. El endofragma y perifragma están en contacto excepto donde el perifragma forma pliegues parasuturales. La forma de la placa antapical es pentagonal y las paraplacas sulcales no fueron observadas. El arqueopilo es epicistal, tipo tAtP. El paracíngulo está limitado por dos crestas paralelas, interrumpidas por una depresión sulcal pronunciada. La ornamentación del perifragma consiste en pústulas de aproximadamente $1 \mu \mathrm{m}$ de diámetro, distribuidas regularmente sobre las áreas intratabulares del quiste.

Dimensiones: Diámetro total máximo en vista polar, 55 $(67,4) 76 \mu \mathrm{m}$; diámetro del cuerpo central en vista polar, 48 $(62,7) 71 \mu \mathrm{m}$; ancho de la cresta cingular, $4(7,1) 10 \mu \mathrm{m}(11$ ejemplares medidos).

Observaciones: Los ejemplares son semejantes a Heteraulacacysta pustulata Jan du Chêne y Adedirán 1985 aunque esta última presenta una ornamentación más pronunciada. H. campanula Drugg y Loeblich 1967 tiene el perifragma ornamentado con gránulos y espinas que pueden fusionarse para formar un microrretículo grosero, además, las crestas cingulares presentan estrías radiales.

\section{Familia Incierta}

Género Batiacasphaera Drugg 1970

Especie tipo: Batiacasphaera compta Drugg 1970.

\section{Batiacasphaera hirsuta Stover 1977 \\ Fig. $12 \mathrm{j}-\mathrm{k}$}

Dimensiones: Largo del cuerpo, 48-50 $\mu \mathrm{m}$; ancho 50-60 $\mu \mathrm{m}$ (2 ejemplares medidos).

Distribución estratigráfica: Según Williams et al. (1998b) se extiende desde el Oligoceno Medio (28,5 Ma) hasta el límite Mioceno Medio/Superior (11,2 Ma). Si bien la mayoría de los registros son del Mioceno medio o más antiguos, esta especie ha sido hallada en depósitos del Mioceno tardío (Poulsen et al., 1996).

\section{Batiacasphaera minuta \\ Matsuoka 1983 emend. Matsuoka y Head 1992}

Fig. $12 \mathrm{a}-\mathrm{c}, \mathrm{f}$

Dimensiones: Diámetro máximo, $32(38,4) 44$ m (19 ejemplares medidos).

Observaciones: Batiacasphaera minuta, B. sphaerica y B. micropapillata son especies muy similares y estrechamente comparables dentro del género. $B$. micropapillata comprende quistes pequeños, esféricos y de acuerdo con Matsuoka y Head (1992), con la superficie de la pared micropapilada; generalmente plegada debido a que la pared es muy delgada. De acuerdo con Stover (1977), B. sphaerica incluye formas con ornamentación más grosera, granulada o reticulada, autoframa más grueso, y conserva su forma esférica o subesférica. B. minuta fue descrita con la superficie del perifragma microrreticulada con muros menores que 5 $\mu \mathrm{m}$ de ancho y lúmenes entre 0,5 y $2 \mu \mathrm{m}$ de diámetro. Los ejemplares hallados en este estudio presentan lúmenes menores de $1 \mu \mathrm{m}$ de diámetro.

\section{Batiacasphaera micropapillata Stover 1977} Fig. $12 \mathrm{~g}-\mathrm{h}$

Dimensiones: Diámetro máximo, 38 (40,2) $44 \mu \mathrm{m}(8$ ejemplares medidos).

\section{Batiacasphaera sphaerica Stover 1977 Fig. $12 \mathrm{~d}-\mathrm{e}$}

Dimensiones: Diámetro máximo $34(35,8) 38 \mu \mathrm{m}$ (7 ejemplares medidos).

Género Cleistosphaeridium (Davey et al. 1966) Eaton et al. 2001

Especie tipo: Cleistosphaeridium diversispinosum (Davey et al. 1966) Eaton et al. 2001.

\section{Cleistosphaeridium ancyreum}

(Cookson y Eisenack 1965) Eaton et al. 2001

Fig. 12 i, l 

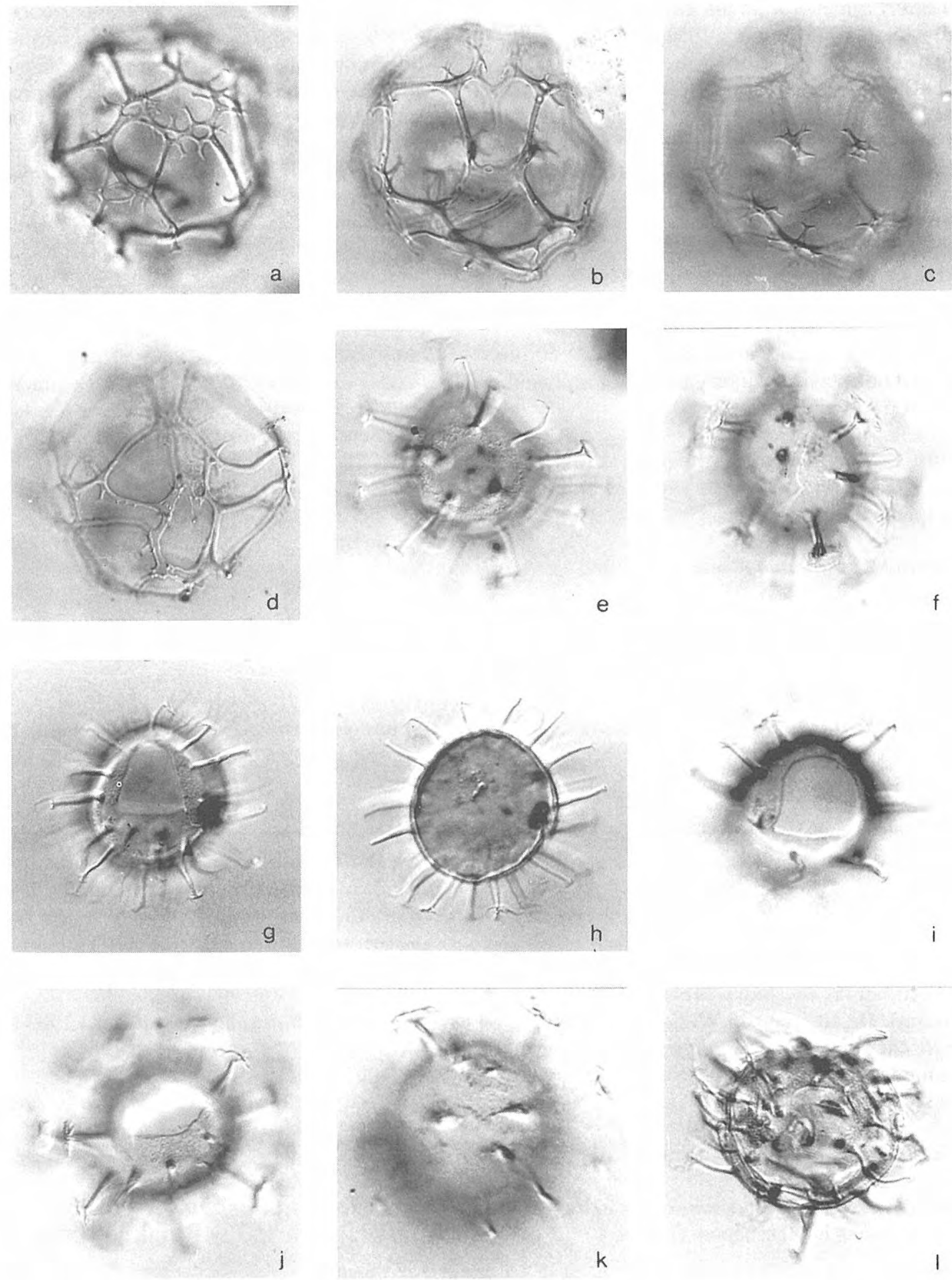

Figura 11. a - d Cannosphaeropsis quattrocchiae Guerstein et al. (x 600); a, b, c, UNSLP 4199 44/99, a, superficie apical, foco bajo, apical surface, low focus; $\mathbf{b}$, superficie antapical, foco intermedio, se observa la placa 1,", , antapical surface, intermediate focus, plate I, ", is observed; c, superficie antapical, foco alto, se observan proyecciones gonales sobre la placa 4"', antapical surface, high focus, showing gonal trifurcate projections on plate 4"',; d, UNSLP 4199 32,2/93,2, superficie ventral, foco alto, ventral surface, high focus. e, Melitasphaeridium choanophorum var. B de Strauss y Lund (x 700), UNSLP 1299 39,7/110. f - i, I Melitasphaeridium spp. (x 700) UNSPL 4199 37/104,5; f, superficie ventral, foco alto, ventral surface, high focus; i, superficie dorsal, foco bajo, dorsal surface, low focus; g, h, (x 900), UNLP 4299 36/97; g, superficie dorsal, foco alto, dorsal surface, high focus; h, corte óptico, cross section; I, (x 750), UNSLP 4900 46,2/95, superficie dorsal, foco alto, dorsal surface, high focus; j, k, Melitasphaeridium choanophorum Harland y Hill UNSPL 1399 46,2/95; j, (x 550), superficie dorsal, foco bajo, dorsal surface, low focus; $\mathbf{k}$, (x 850), superficie ventral, foco alto, ventral surface, high focus. 
Observaciones: La mayoría de los ejemplares presentan procesos con los extremos asimétricamente bifurcados, en forma de piqueta ("dolobrate"). Este rasgo morfológico diferencia el género Cleistosphaeridium del género jurásico Systematophora (Eaton et al., 2001). Los ejemplares bien conservados, orientados en vista dorsoventral, exhiben una forma asimétrica lenticular y la depresión sulcal desplazada hacia la izquierda. Eaton et al. (2001) incluyen a $C$. ancyreum en el complejo diversispinosum, caracterizado por estos dos últimos aspectos que sugieren una posible afinidad con la familia Areoligeraceae.

Dimensiones: Ancho del cuerpo central $43(51,4) 60 \mu \mathrm{m}$, largo cuerpo central $34(42,3) 58 \mu \mathrm{m}$, largo de los procesos $10(12,7) 20 \mu \mathrm{m}$ (18 ejemplares medidos).

Distribución estratigráfica: La distribución de $C$. ancyreum se extiende desde el Eoceno temprano al Mioceno medio (Eaton et al., 2001).

\section{Género Dapsilidinium Bujak et al. 1980}

Especie tipo: Dapsilidinium pastielsii (Davey y Williams 1966) Bujak et al. 1980.

\section{Dapsilidinium pseudocolligerum (Stover 1977) Bujak et al., 1980} Fig. $6 \mathrm{a}-\mathrm{c}$

Dimensiones: Diámetro del cuerpo central, 31 (36,3) $40 \mu \mathrm{m}$; largo de los procesos, $9(12,9) 18 \mu \mathrm{m}$ (21 ejemplares medidos).

Distribución estratigráfica: Según De Verteuil (1996) el registro más alto de Dapsilidiniun pseudocolligerun en la plataforma continental de Nueva Jersey corresponde al Mioceno terminal. De acuerdo con Williams et al. (1998b), esta especie se encuentra desde el Eoceno medio - superior (41,3 Ma) hasta el Mioceno superior, en el límite Tortoniense - Serravaliense (7,1 Ma).

\section{Género Distatodinium Eaton 1976}

Especie tipo: Distatodinium craterum Eaton 1976 emend. Zevenboom y Santarelli en Zevenboom (1995).

\section{Distatodinium paradoxum (Brosius 1963) Eaton 1976}

Fig. 4 d - e

Dimensiones: Ancho del cuerpo del quiste 27 (30) $33 \mu \mathrm{m}$, largo del cuerpo del quiste $45(53,3) 60 \mu \mathrm{m}$, largo de los procesos $18(24,2) 30 \mu \mathrm{m}$ (13 ejemplares medidos).

Registros previos: Según Bujak y Matsuoka (1986) D. paradoxum desaparece junto a otras varias especies en el Mioceno medio temprano en el Norte de Japón. El registro más alto de $D$. paradoxum caracteriza al techo de la Zona Labyrinthodinium truncatum asignada al Mioceno medio, en el margen este de la cuenca del Mar del Norte y en sedimentos del Mar de Noruega (Piasecki, 1980; Manum et al., 1989). Head et al. (1989c) registran la última presencia de esta especie en la Zona de Asociación BB3 de edad Mioceno ? medio. De acuerdo con Powell (1992) D. paradoxum tiene la última aparición en la Zona de Intervalo Labyrinthodinium truncatum (Ltr) cuya edad fue asignada al intervalo Langhiense-Serravaliense (parte), Mioceno medio (parte). El techo de la Zona Mio3, equivalente a una edad langhiense-serravaliense en sedimentos del Mar de Groenlandia, está indicado por el registro más alto de $D$. paradoxum (Poulsen et al., 1996). Según Williams et al. (1998b) esta especie se encuentra desde los 41,9 $\mathrm{Ma}$ (Luteciense) hasta los 13,6 Ma. (Serravaliense).

\section{Género Labyrinthodinium Piasecki 1980}

Especie tipo: Labyrinthodinium truncatum Piasecki 1980.

\section{Labyrinthodinium truncatum subsp. truncatum Piasecki 1980}

Fig. $6 \mathrm{~d}-\mathrm{f}$

Dimensiones: Diámetro del cuerpo central, 21 (24) $30 \mu \mathrm{m}$; largo de los procesos 4,5 $(5,4) 7 \mu \mathrm{m}$ (18 ejemplares medidos).

Registros previos: Por su corto biocrón es un importante indicador bioestratigráfico; los registros corresponden al intervalo Mioceno Temprano tardío? - Mioceno Tardío temprano (Head et al, 1989a) y según Williams et al. (1998b), se extiende desde el Burdigaliense (17,4 Ma) al Tortoniense (7,8 Ma).

Género Reticulatosphaera Matsuoka 1983 emend. Bujak y Matsuoka 1986

Especie tipo: Reticulatosphaera actinocoronata (Benedek 1972) Bujak y Matsuoka 1986.

\section{Reticulatosphaera actinocoronata (Benedek 1972) Bujak y Matsuoka 1986 Fig. $6 \mathrm{~g}-\mathrm{h}$}

Dimensiones: Diámetro del cuerpo central, 22 (24,8) 30 $\mu \mathrm{m}$; diámetro total, 38 (44) $50 \mu \mathrm{m}$; largo de los procesos 9 $(10,6) 12 \mu \mathrm{m}$ (14 ejemplares medidos).

Observaciones: Todos los ejemplares hallados presentan el cuerpo central deformado y, como en la mayoría de los registros de esta especie, el arqueopilo es difícilmente observable. 

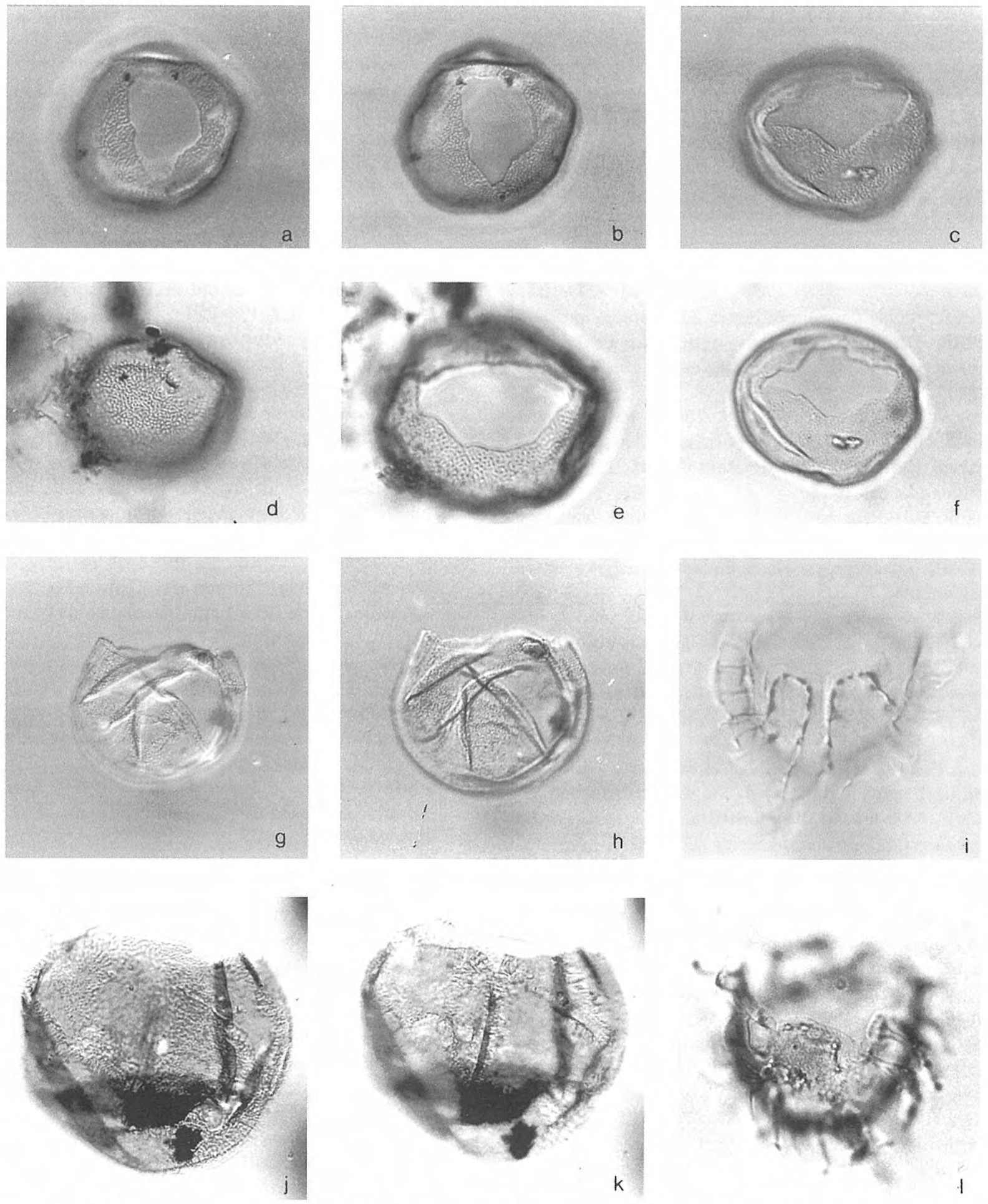

Figura 12. a - c, f Batiacasphaera minuta (Matsuoka) Matsuoka y Head, (x 800). a, b, UNSLP 4999 23/102, superficie apical, foco bajo, apical surface, low focus; c, f, UNSLP 4299 29,5/92,2, vista apical oblicua, foco alto, oblique apical view, high focus. d, e Batiacasphaera sphaerica Stover, UNSLP 1199 27,6/101; d (x 750), superficie antapical, foco bajo, antapical surface, low focus; e, (x 1000), superficie apical, foco alto, apical surface, high focus. g, h Batiacasphaera micropapillata Stover, UNSLP 4799 38,5/111, vista lateral, lateral view; g, (x 650); h, (x 850). i, l Cleistosphaeridium ancyreum (Cookson y Eisenack) Eaton (x 500); i, UNSLP 5099 24,5/105, superficie dorsal, foco alto, dorsal surface, high focus; I, UNSLP 1899 32/102,5, superficie ventral, foco alto, se observa la depresión sulcal desplazada hacia la izquierda, ventral surface, high focus, showing the sulcal notch offset to the left. j, k Batiacasphaera hirsuta Stover (x 750), UNSLP 2299 34,5/104, vista lateral, lateral view; j, foco alto, high focus; $\mathbf{k}$, foco bajo, low focus. 


\section{AGRADECIMIENTOS}

Este trabajo fue financiado por el proyecto FONCYT PICT 07-09659. Las autoras agradecen al Departamento de Exploración de YPF que cedieron las muestras y al $\mathrm{Sr}$. Orlando Cárdenas por el procesamiento de las mismas. Asimismo, se agradece a los Dres. M. Head y J. Wrenn por la discusión de alguna de las especies descritas en este trabajo.

\section{BIBLIOGRAFÍA}

Las referencias correspondientes a la nomenclatura de quistes de dinoflagelados se encuentran citadas en Fensome et al. (1993) y en Williams et al. (1998a).

Barreda, V. D. y Palamarczuk, S. 2000a. Palinomorfos continentales y marinos de la Formación Monte León en su área tipo, provincia de Santa Cruz, Argentina. Ameghiniana, 37, 3-12.

Barreda, V. D. y Palamarczuk, S. 2000b. Palinoestratigrafía de depósitos del Oligoceno tardío-Mioceno en el área sur del Golfo San Jorge, provincia de Santa Cruz, Argentina. Ameghiniana, 37, 103-117.

Berggren, W.A., Kent, D.V., Swisher, III, C.C., and Aubry, M.-P. 1995. A revised Cenozoic geochronology and chronostratigraphy. In: Geochronology, Time scales and Global Stratigraphic Correlation (Eds. W.A. Berggren, D.V. Kent, M.P. Aubry and J. Hardenbol). SEPM Special Publication, 54, 129-212.

Bujak, J.P. and Matsuoka, K. 1986. Late Cenozoic dinoflagellate cyst zonation in the western and northern Pacific. American Association of Stratigraphic Palynologists Contribution Series, 17, 7-25.

Bujak, J.P., Downie, C., Eaton, G.L. and Williams, G.L. 1980. Taxonomy of some Eocene dinoflagellate cyst species from southern England. In: Bujak, J.P., Downie, C., Eaton, G.L. and Williams, G.L. Dinoflagellate Cysts and Acritarchs from the Eocene of Southern England. The Palaeontological Association, Special Papers in Palaeontology, 24, 26-36.

De Verteuil, L. 1996. Data report: Upper Cenozoic dinoflagellate cysts from the Continental Slope and Rise off New Jersey. In: Proceedings of the Ocen Drilling Program, Scientific Results, 150 (Eds. G.S. Mountain, K.G. Miller, P. Blum, C.W. Poag and D.C. Twichell), 439-454.

De Verteuil, L. and Norris, G. 1996. Miocene dinoflagellate stratigraphy and systematics of Maryland and Virginia. Micropaleontology, 42, Supplement I-VIII, 72 pp.

Eaton, G.L, Fensome, R.A, Riding, J.B. and Williams, G.L. 2001. Re-evaluation of the status of the dinoflagellate cyst genus Cleistosphaeridium. Neues Jahrbuch für Geologie und Paläontologie Abhandlungen, 219, 171-205.

Edwards L.E. and Andrle, V.A.S. 1992. Distribution of selected dinoflagellate cysts in modern marine sediments. In: Neogene and Quaternary dinoflagellate cysts and acritarchs (Eds. M.J. Head and J.H. Wrenn). American Association of Stratigraphic Palynologists Foundation, 259-288.
Fensome, R. A., Taylor, F.J.R., Norris, G., Sarjeant, W.A.S., Wharton, D.I. and Williams, G.L. 1993. A classification of fossil and living dinoflagellates. Micropaleontology Press Special Paper, 7, 351 pp.

Fensome, R.A., MacRae, R.A. and Williams, G.L. 1998. DINOFLAJ. Geological Survey of Canada, Open File D3653. Dartmouth, Nova Scotia.

Fryklund, R., Marshall, A. y Steves, J. 1996. La Cuenca del Colorado. In: Geología y Recursos Naturales de la Plataforma Continental Argentina (Eds. V.A. Ramos y M.A. Turic) XIII Congreso Geológico Argentino y III Congreso de Exploración de Hidrocarburos, Buenos Aires. Relatorio 8, 135-158.

Gamerro, J. C. y Archangelsky, S. 1981. Palinozonas neocretácicas y terciarias de la Plataforma Continental Argentina en la Cuenca del Colorado. Revista Española de Micropaleontología, 13, 110-140.

Guerstein, G. R. 1990a. Palinología estratigráfica del Terciario de la Cuenca del Colorado, República Argentina. Parte II: especies marinas de la perforación Nadir No. 1. Revista Española de Micropaleontología, 22, 167-182.

Guerstein, G. R. 1990b. Palinología estratigráfica del Terciario de la Cuenca del Colorado, República Argentina. Parte III: estudio sistemático de la perforación Puerto Belgrano No 20. Revista Española de Micropaleontología, 22, 459-480.

Guerstein, G. R. y Guler, M. V. 2000. Bioestratigrafía en base a quistes de dinoflagelados del Eoceno-Mioceno del Pozo (YPF) Ombucta x-1. Cuenca del Colorado, Argentina. Ameghiniana, 37, 81-90.

Guerstein, G.R. y Junciel, G.L. 2001. Quistes de dinoflagelados del Cenozoico de la Cuenca del Colorado, Argentina. Ameghiniana, 38, 299-316.

Guerstein, G.R., Williams, G.L. and Fensome, R.A. 2001. Cannosphaeropsis quattrocchiae, a new species of dinoflagellate cyst from the mid cenozoic of the Colorado Basin, Argentina. Micropaleontology, 47, 155-167.

Guler, M.V., (en prensa). Quistes de dinoflagelados de la Familia Protoperidiniaceae del Neógeno de la cuenca del Colorado. Ameghiniana.

Guler, M.V. y Guerstein, G.R. (en prensa). Bioestratigrafía del Oligoceno-Mioceno de la cuenca del Colorado, basada en quistes de dinoflagelados. Revista Española de Micropaleontología.

Guler, M.V, Guerstein, G.R. y Malumián, N. 2002. Biostratigrafía de la Formación Barranca Final, Neógeno de la cuenca del Colorado, Argentina. Ameghiniana, 39, 103-110.

Harland, R. 1983. Distribution maps of recent dinoflagellate cysts in bottom sediments from the north Atlantic ocean and adjacent seas. Palaeontology, 26, 321-387.

Harland, R. and Hill, J. 1979. A reappraisal of the Cenozoic dinoflagellate cyst "Hystrichosphaeridium" choanophorum Deflandre and Cookson 1955. Review of Palaeontology and Palynology, 28, 37-45.

Head, M.H. 1994a. Morphology and paleoenvironmental significance of the Cenozoic dinoflagellates genera Tectadonium and Habibacysta. Micropaleontology, 40, 289-321. 
Head, M.H. 1994b. A forum on Neogene and Quaternary dinoflagellate cysts. Third Workshop on Neogene and Quaternary dinoflagellates. Palynology, 17, 201-240.

Head, M. J. 1996. Late Cenozoic dinoflagellates from the Royal Society borehole at Ludham, Norfolk, eastern England. Journal of Paleontology, 70, 543-570.

Head, M.J. 1997. Thermophilic dinoflagellate assemblages from the Mid Pliocene of Eastern England. Journal of Paleontology, 71, 165-193.

Head, M.J. 1998. Marine environmental change in the Pliocene and early Pleistocene of eastern England: the dinoflagellate evidence reviewed. Mededelingen Nederlands Instituut voor Toegepaste Geowetenschappen TNO 60, 199-226.

Head, M. J. and Westphal, H. 1999. Palynology and Paleoenvironments of a Pliocene carbonate platform: The Clino Core, Bahamas. Journal of Paleontology, 73, 1-25.

Head, M.J., Norris, G. and Mudie, P.J. 1989a. Palynology and dinocyst stratigraphy of the Upper Miocene ad lowermost Pliocene, ODP Leg 105, Site 646, Labrador Sea. In: Proceedings of the Ocean Drilling Program, Scientific Results, 105 (Eds. S.P. Srivastava, M. Arthur and B. Clement), 423-451.

Head, M.J., Norris, G. and Mudie, P.J. 1989b. New species of dinocysts and a new species of acritarch from the upper Miocene and lowermost Pliocene, ODP Leg 105, Site 646, Labrador Sea. In: Proceedings of the Ocean Drilling Program, Scientific Results, 105 (Eds. S.P. Srivastava, M. Arthur and B. Clement), 453-466.

Head, M.J., Norris, G. and Mudie, P.J. 1989c. Palynology and dinocyst stratigraphy of the Miocene in ODP Leg 105, Hole 645E, Baffin Bay. In: Proceedings of the Ocean Drilling Program, Scientific Results, 105 (Eds. S.P. Srivastava, M. Arthur and B. Clement), 467-514.

Lentin, J.K. and Williams, G.L. 1985. Fossil dinoflagellates: index to genera and species, 1985 edition. Canadian Tecnical Report of Hydrography and Ocean Sciences, 60, $451 \mathrm{pp}$.

Manum, S.B., Boulter, M.C., Gunnarsdottir, H., Rangnes, K. and Scholze, A. 1989. Eocene to Miocene palynology of the Norwegian Sea (ODP Leg 104). Proceedings of the Ocean Drilling Program, Scientific Results, 104, 611-662.

Matsuoka, K. and Head, M.J. 1992. Taxonomic revision of the Neogene marine palynomorphs Cyclopsiella granosa (Matsuoka) and Batiacasphaera minuta (Matsuoka), and a new species of Pyxidinopsis Habib (Dinophyceae) from the Miocene of the Labrador Sea. In: Neogene and Quaternary Dinoflagellate Cysts and Acritarchs (Eds. M.J. Head and J.H. Wrenn). American Association of Stratigraphic Palynologists Foundation, 165-180.

McMinn, A. 1991. Recent dinoflagellate cysts from estuaries on the central coast of New South Wales, Australia. Micropaleontology, 37, 269-287.

Palamarczuk, S. y Barreda, V.D. 1998. Bioestratigrafía en base a quistes de dinoflagelados de la Formación Chenque (Mioceno), Provincia del Chubut, Argentina. Ameghiniana, 35, 415-126.

Palamarczuk, S. y Barreda, V. 2000. Palinología del Paleógeno tardío-Neógeno temprano, pozo Aries x-1, Plataforma Continental Argentina, Tierra del Fuego.
Ameghiniana, 37, 221-234.

Piasecki, S. 1980. Dinoflagellate cysts stratigraphy of the Miocene Hodde and Gram Formation, Denmark. Bulletin of the Geological Society of Denmark, 29, 53-76.

Poulsen, N. E, Manum, S.G, Williams, G.L and Ellegaard, M. 1996. Tertiary dinoflagellate biostratigraphy of sites 907, 908 and 909 in the Norwegian-Greenland Sea. Proceedings of the Ocean Drilling Program, Scientific Results 151, 255-287.

Powell, A.J. 1992. Dinoflagellate cysts of the Tertiary System. In: A stratigraphic index of dinoflagellate cysts (Ed. A.J. Powell). Chapman and Hall, London, 155-251.

Rochon, A., Vernal, A. de, Turon, J.L, Matthiessen J. and Head, M.J. 1999. Distribution of recent dinoflagellate cysts in surface sediments from the North Atlantic Ocean and adjacent seas in relation to sea-surface parameters. American Association of Stratigraphic Palynologists, Contribution Series 35, 152 pp.

Stover, L.E. 1977. Oligocene and early Miocene dinoflagellates from Atlantic Corehole 5/5B, Blake Plateau. In: American Association of Stratigraphic Palynologists Contribution Series 5 (Ed. W.C. Elsid), 66-90.

Strauss, C. and Lund, J.J. 1992. A middle Miocene dinoflagellate cyst microflora from Papendorf near Hamburg, Germany. Mitteilungen GeologischPaläontologischen Institut der Universität Hamburg, 73, 159-189.

Wall, D., Dale, B., Lohman, G.P. and Smith, W.K. 1977. The environmental and climatic distribution of dinoflagellate cysts in modern marine sediments from regions in the North and South Atlantic oceans and adjacent seas. Marine Micropaleontology, 2, 121-200.

Warny, S.A and Wrenn, J.H 1997. New species of dinoflagellate cysts from the Bou Regreg Core: a Miocene Pliocene boundary section on the Atlantic Coast of Morocco. Review of Palaeobotany and Palynology, 96, 281-304

Williams, G.L., Lentin, J. and Fensome, R.A. 1998a. The Lentin and Williams Index of fossil dinoflagellates 1998 edition. American Association of Stratigraphic Palynologist Contribution Series 34, 817 pp. Dallas.

Williams, G.L., Brinkhuis, H., Bujak, J.P., Damassa, S.P., Hochuli, P.A., De Verteuil, L. and Zevenboom, D. 1998b. Dinoflagellates. In: Appendix to Mesozoic and Cenozoic sequence chronostratigraphic framework of European Basins. Hardenbol, J., Thierry, J., Farley, M.B., Jacquin, T., Graciansky, P-C. de, and Vail, P.R. In Mesozoic and Cenozoic Sequenc Stratigraphy of European Basins. Society for Sedimentary Geology, Special Publication 60, p. 9.

Wrenn, J.H. 1988. Differentiating species of Nemmatosphaeropsis Cookson and Eisenack 1955. Palynology, 12, 129-150.

Zevenboom, D. 1995. Dinoflagellate cysts from the Mediterranean Late Oligocene and Miocene. Tesis Doctoral, Universidad de Utrech (inédita), 221 pp.

Manuscrito recibido: 20 de febrero, 2002 Manuscrito aceptado: 23 de septiembre, 2002 